\title{
Sequential Pd- and Rh-Catalyzed Three-Component Cyclization with Allenylboronate Platform
}

\author{
Keisuke Tonogaki, ${ }^{+}$Kenichiro Itami, ${ }^{*}$, and Jun-ichi Yoshida*,+ \\ 'Department of Synthetic Chemistry \& Biological Chemistry, Graduate School of Engineering, \\ Kyoto University, Nishikyo-ku, Kyoto 615-8510, Japan \\ ‡Research Center for Materials Science, Nagoya University, Nagoya 464-8602, Japan
}

General. ${ }^{1} \mathrm{H}$ and ${ }^{13} \mathrm{C}$ NMR spectra were recorded on Varian MERCURY plus-400 $\left({ }^{1} \mathrm{H} 400 \mathrm{MHz},{ }^{13} \mathrm{C}\right.$ $100 \mathrm{MHz})$ spectrometer in $\mathrm{CDCl}_{3}$ using tetramethylsilane $\left({ }^{1} \mathrm{H}, \delta=0.00 \mathrm{ppm}\right)$ and $\mathrm{CDCl}_{3}\left({ }^{13} \mathrm{C}, \delta=77.0\right.$ ppm) as internal standards or in $\mathrm{C}_{6} \mathrm{D}_{6}$ using $\mathrm{C}_{6} \mathrm{D}_{6}\left({ }^{1} \mathrm{H}, \delta=7.15 \mathrm{ppm},{ }^{13} \mathrm{C}, \delta=128.02 \mathrm{ppm}\right)$ as internal standards. EI mass spectra were recorded on JMS-SX102A spectrometer. Gel permeation chromatography was carried out with Japan Analytical Industry LC-918. Unless otherwise noted, all reactions were carried out under argon atmosphere. Column chromatography was performed with silica gel 60 A (SILICYCLE). Unless otherwise noted, all materials were obtained from commercial suppliers and used without further purification. Phenylallene ${ }^{1}$ and triisopropylsilylallene ${ }^{2}$ were prepared by the literature procedures. Allenylboronate pinacol ester (1) was prepared by the literature procedure. $^{3}$

Typical Procedure for Three-Component Assembly of Allenylboronate Pinacol Ester (1), Carbon Nucleophiles (2), and Aryl Iodides (3) (Table 1). A mixture of diethyl methylmalonate (104.5 mg, 0.60 $\mathrm{mmol}$ ) and sodium hydride (55\% suspension in mineral oil, $26 \mathrm{mg}, 0.60 \mathrm{mmol})$ in dry toluene $(1.0 \mathrm{~mL})$ was stirred at room temperature for $1 \mathrm{~h}$ under argon. To this suspension were added 4 -iodotoluene (3a; $64.6 \mathrm{mg}, 0.30 \mathrm{mmol})$, allenylboronate pinacol ester $(\mathbf{1} ; 99.0 \mathrm{mg}, 0.60 \mathrm{mmol})$, and a solution of $\mathrm{Pd}_{2}(\mathrm{dba})_{3}$ (6.9 mg, $7.5 \mu \mathrm{mol}, 2.5 \mathrm{~mol} \%)$ and $\mathrm{P}_{\left(\mathrm{C}_{6} \mathrm{H}_{4} \mathrm{CF}_{3}-4\right) 3}(14.0 \mathrm{mg}, 30 \mu \mathrm{mol}, 10 \mathrm{~mol} \%)$ in dry toluene $(0.5 \mathrm{~mL})$, and the resultant mixture was stirred at $80{ }^{\circ} \mathrm{C}$ for $24 \mathrm{~h}$. After cooling the reaction mixture to room temperature, the catalyst and salt were removed by filtration through a short silica gel pad (EtOAc). The filtrate was evaporated and the residue was subjected to silica gel chromatography (hexane/EtOAc $=15 / 1$ to $10 / 1)$ to afford 4 aa $(75.5 \mathrm{mg}, 59 \%)$ as colorless oil.

4aa: 59\% yield from 1, sodium salt of diethyl methylmalonate (2a), and 4-iodotoluene (3a). ${ }^{1} \mathrm{H}$ NMR $\left(400 \mathrm{MHz}^{\mathrm{CDCl}} \mathrm{CD}_{3} \delta 1.11(\mathrm{t}, J=7.2 \mathrm{~Hz}, 6 \mathrm{H}), 1.29(\mathrm{~s}, 12 \mathrm{H}), 1.35(\mathrm{~s}, 3 \mathrm{H}), 2.29(\mathrm{~s}, 3 \mathrm{H}), 3.59(\mathrm{~s}, 2 \mathrm{H}), 3.66-3.74\right.$ $(\mathrm{m}, 2 \mathrm{H}), 3.78-3.86(\mathrm{~m}, 2 \mathrm{H}), 5.61(\mathrm{~s}, 1 \mathrm{H}), 7.05(\mathrm{~d}, J=8.0 \mathrm{~Hz}, 2 \mathrm{H}), 7.19(\mathrm{~d}, J=8.0 \mathrm{~Hz}, 2 \mathrm{H}) .{ }^{13} \mathrm{C}$ NMR $(100$ $\left.\mathrm{MHz}_{\mathrm{CDCl}}\right) \delta 13.8,19.1,21.1,24.9,37.6,52.8,60.8,83.0,126.7,128.1,137.2,140.6,158.2,171.5$. HRMS (EI) $\mathrm{m} / \mathrm{z}$ calcd for $\mathrm{C}_{24} \mathrm{H}_{35} \mathrm{BO}_{6}: 430.2527$, found 430.2526 .

The stereochemistry of 4 aa was determined by the NOESY experiments. Representative results are shown below.

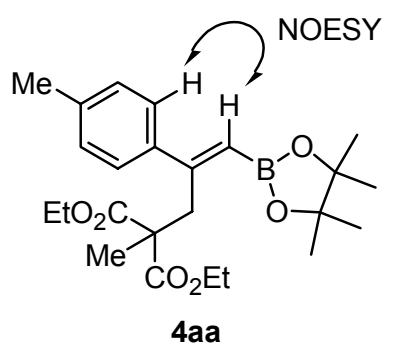


4ab: $64 \%$ yield from 1 , sodium salt of diethyl methylmalonate (2a), and iodobenzene $(3 \mathbf{b}) .{ }^{1} \mathrm{H}$ NMR $\left(400 \mathrm{MHz}, \mathrm{CDCl}_{3}\right) \delta 1.09(\mathrm{t}, J=7.2 \mathrm{~Hz}, 6 \mathrm{H}), 1.29(\mathrm{~s}, 12 \mathrm{H}), 1.36(\mathrm{~s}, 3 \mathrm{H}), 3.60(\mathrm{~s}, 2 \mathrm{H}), 3.62-3.70(\mathrm{~m}, 2 \mathrm{H})$, 3.76-3.84 (m, 2H), $5.62(\mathrm{~s}, 1 \mathrm{H}), 7.17-7.29(\mathrm{~m}, 5 \mathrm{H}) .{ }^{13} \mathrm{C} \mathrm{NMR}\left(100 \mathrm{MHz}, \mathrm{CDCl}_{3}\right) \delta$ 13.9, 19.2, 24.9, 37.7, 52.8, $60.9,83.1,126.9,127.51,127.54,143.6,158.2,171.6$. HRMS (EI) $\mathrm{m} / z$ calcd for $\mathrm{C}_{23} \mathrm{H}_{33} \mathrm{BO}$ : 416.2370 , found 416.2372.

4ac: $54 \%$ yield from 1 , sodium salt of diethyl methylmalonate $(2 \mathrm{a})$, and 4-iodobenzonitrile $(3 \mathrm{c}) .{ }^{1} \mathrm{H}$ NMR $\left(400 \mathrm{MHz}, \mathrm{CDCl}_{3}\right) \delta 1.11(\mathrm{t}, J=6.8 \mathrm{~Hz}, 6 \mathrm{H}), 1.30(\mathrm{~s}, 12 \mathrm{H}), 1.35(\mathrm{~s}, 3 \mathrm{H}), 3.57(\mathrm{~s}, 2 \mathrm{H}), 3.65-3.73(\mathrm{~m}, 2 \mathrm{H})$, 3.79-3.87 (m, 2H), $5.66(\mathrm{~s}, 1 \mathrm{H}), 7.38(\mathrm{~d}, J=8.4 \mathrm{~Hz}, 2 \mathrm{H}), 7.54(\mathrm{~d}, J=8.4 \mathrm{~Hz}, 2 \mathrm{H}) .{ }^{13} \mathrm{C}$ NMR $(100 \mathrm{MHz}$, $\left.\mathrm{CDCl}_{3}\right) \delta 13.9,19.2,24.9,37.5,52.8,61.1,83.5,111.1,118.6,127.6,131.4,148.3,156.0,171.3$. HRMS (EI) $\mathrm{m} / z$ calcd for $\mathrm{C}_{24} \mathrm{H}_{32} \mathrm{BNO}_{6}$ : 441.2323, found 441.2325.

4bb: $66 \%$ yield from 1 , sodium salt of ethyl 2-methylacetoacetate $(2 \mathrm{~b})$, and iodobenzene $(3 \mathrm{~b}) .{ }^{1} \mathrm{H}$ NMR (400 MHz, CDCl $) \delta 1.03(\mathrm{t}, J=7.2 \mathrm{~Hz}, 3 \mathrm{H}), 1.275(\mathrm{~s}, 3 \mathrm{H}), 1.280(\mathrm{~s}, 6 \mathrm{H}), 1.29(\mathrm{~s}, 6 \mathrm{H}), 2.05(\mathrm{~s}, 3 \mathrm{H})$, 3.32-3.40 (m, 1H), $3.43(\mathrm{~d}, J=13.6 \mathrm{~Hz}, 1 \mathrm{H}), 3.59-3.65(\mathrm{~m}, 1 \mathrm{H}), 3.65(\mathrm{~d}, J=13.6 \mathrm{~Hz}, 1 \mathrm{H}), 5.60(\mathrm{~s}, 1 \mathrm{H})$, 7.19-7.28 (m, 5H). ${ }^{13} \mathrm{C}$ NMR (100 MHz, $\left.\mathrm{CDCl}_{3}\right) \delta$ 13.7, 18.3, 24.9, 25.0, 25.9, 37.1, 59.0, 60.9, 83.1, 126.9, 127.6, 143.7, 158.7, 171.8, 204.8. HRMS (EI) $m / z$ calcd for $\mathrm{C}_{22} \mathrm{H}_{31} \mathrm{BO}_{5}: 386.2265$, found 386.2265.

4cd: $67 \%$ yield from 1, sodium salt of ethyl 2-oxocyclohexanecarboxylate (2c), and 3-iodothiophene (3d). ${ }^{1} \mathrm{H}$ NMR $\left(400 \mathrm{MHz}, \mathrm{CDCl}_{3}\right) \delta 1.07(\mathrm{t}, J=6.8 \mathrm{~Hz}, 3 \mathrm{H}), 1.26(\mathrm{~s}, 6 \mathrm{H}), 1.27(\mathrm{~s}, 6 \mathrm{H}), 1.53-1.70(\mathrm{~m}, 4 \mathrm{H})$, 1.95-2.01 (m, 1H), 2.26-2.35 (m, 1H), 2.43-2.48 (m, 2H), $3.37(\mathrm{~d}, J=13.6 \mathrm{~Hz}, 1 \mathrm{H}), 3.45(\mathrm{~d}, J=13.6 \mathrm{~Hz}, 1 \mathrm{H})$, 3.51-3.66 (m, 2H), $5.74(\mathrm{~s}, 1 \mathrm{H}), 7.12(\mathrm{dd}, J=4.8,1.6 \mathrm{~Hz}, 1 \mathrm{H}), 7.15-7.18(\mathrm{~m}, 2 \mathrm{H}) .{ }^{13} \mathrm{C}$ NMR $(100 \mathrm{MHz}$, $\left.\mathrm{CDCl}_{3}\right) \delta 13.8,22.7,24.9,25.0,27.8,34.9,37.2,41.1,59.9,60.9,83.0,121.7,124.9,126.5,145.1,152.1,170.4$, 206.5. HRMS (EI) $\mathrm{m} / \mathrm{z}$ calcd for $\mathrm{C}_{22} \mathrm{H}_{31} \mathrm{BO}_{5} \mathrm{~S}: 418.1985$, found 418.1983 .

4db: $67 \%$ yield from 1, sodium salt of 2-cyanopropionic acid ethyl ester (2d), and iodobenzene (3b). ${ }^{1} \mathrm{H}$ NMR $\left(400 \mathrm{MHz}, \mathrm{CDCl}_{3}\right) \delta 1.06(\mathrm{t}, J=7.2 \mathrm{~Hz}, 3 \mathrm{H}), 1.32(\mathrm{~s}, 12 \mathrm{H}), 1.55(\mathrm{~s}, 3 \mathrm{H}), 3.46-3.54(\mathrm{~m}, 1 \mathrm{H}), 3.47(\mathrm{~d}$, $J=13.2 \mathrm{~Hz}, 1 \mathrm{H}), 3.64(\mathrm{~d}, J=13.2 \mathrm{~Hz}, 1 \mathrm{H}), 3.71-3.79(\mathrm{~m}, 1 \mathrm{H}), 5.80(\mathrm{~s}, 1 \mathrm{H}), 7.25-7.36(\mathrm{~m}, 5 \mathrm{H}) .{ }^{13} \mathrm{C}$ NMR $(100$ $\left.\mathrm{MHz}_{2} \mathrm{CDCl}_{3}\right) \delta 13.7,23.8,25.0,40.3,44.2,62.4,83.4,119.2,126.7,128.0,128.2,142.3,156.0,168.9$. HRMS (EI) $\mathrm{m} / \mathrm{z}$ calcd for $\mathrm{C}_{21} \mathrm{H}_{28} \mathrm{BNO}_{4}: 369.2111$, found 369.2111 .

4ee: $54 \%$ yield from 1 , sodium salt of diethylmalonate (2e), and 4-chloroiodobenzene (3e). ${ }^{1} \mathrm{H}$ NMR $\left(400 \mathrm{MHz}, \mathrm{CDCl}_{3}\right) \delta 1.17(\mathrm{t}, J=6.8 \mathrm{~Hz}, 6 \mathrm{H}), 1.30(\mathrm{~s}, 12 \mathrm{H}), 3.38(\mathrm{dd}, J=8.4,6.4 \mathrm{~Hz}, 1 \mathrm{H}), 3.45-3.47(\mathrm{~m}, 2 \mathrm{H})$, 4.00-4.08 (m, 4H), $5.66(\mathrm{~s}, 1 \mathrm{H}), 7.25-7.31(\mathrm{~m}, 4 \mathrm{H}) .{ }^{13} \mathrm{C} \mathrm{NMR}\left(100 \mathrm{MHz}, \mathrm{CDCl}_{3}\right) \delta$ 14.1, 25.0, 32.4, 52.0, 61.2, 83.3, 127.7, 128.3, 133.8, 140.8, 157.2, 168.6. HRMS (EI) $\mathrm{m} / \mathrm{z}$ calcd for $\mathrm{C}_{22} \mathrm{H}_{30} \mathrm{BClO} 6$ : 436.1824 , found 436.1824 .

4fa: $69 \%$ yield from 1, sodium salt of cyanoacetic acid ethyl ester (2f), and 4-iodotoluene (3a). ${ }^{1} \mathrm{H}$ $\operatorname{NMR}\left(400 \mathrm{MHz}, \mathrm{CDCl}_{3}\right) \delta 1.25(\mathrm{t}, J=6.8 \mathrm{~Hz}, 3 \mathrm{H}), 1.32(\mathrm{~s}, 12 \mathrm{H}), 2.34(\mathrm{~s}, 3 \mathrm{H}), 3.43(\mathrm{dd}, J=13.2,6.0 \mathrm{~Hz}, 1 \mathrm{H})$, $3.53(\mathrm{dd}, J=8.8,6.0 \mathrm{~Hz}, 1 \mathrm{H}), 3.69(\mathrm{dd}, J=13.2,8.8 \mathrm{~Hz}, 1 \mathrm{H}), 4.13(\mathrm{q}, J=6.8 \mathrm{~Hz}, 2 \mathrm{H}), 5.84(\mathrm{~s}, 1 \mathrm{H}), 7.14(\mathrm{~d}, J$ $=8.0 \mathrm{~Hz}, 2 \mathrm{H}), 7.30(\mathrm{~d}, J=8.0 \mathrm{~Hz}, 2 \mathrm{H}) .{ }^{13} \mathrm{C} \mathrm{NMR}\left(100 \mathrm{MHz}, \mathrm{CDCl}_{3}\right) \delta 14.0,21.2,24.9,25.0,33.0,37.7,62.6$, 83.4, 115.8, 126.2, 129.2, 137.9, 138.5, 155.8, 165.7. HRMS (EI) $\mathrm{m} / \mathrm{z}$ calcd for $\mathrm{C}_{21} \mathrm{H}_{28} \mathrm{BNO}_{4}$ : 369.2111, found 369.2108 .

2-Methyl-2-(3-phenyl-2-p-tolyl-allyl)malonic acid diethyl ester (Scheme 4): ${ }^{1} \mathrm{H}$ NMR (400 MHz, $\left.\mathrm{CDCl}_{3}\right)$ This compound was obtained as a mixture of isomers $(E / Z=48 / 52) .{ }^{1} \mathrm{H}$ NMR $\left(400 \mathrm{MHz}, \mathrm{CDCl}_{3}\right)$ $\delta 1.06(\mathrm{t}, J=6.8 \mathrm{~Hz}, 6 \mathrm{H}, E$-isomer), 1.12 (s, 3H, E-isomer), $1.14(\mathrm{t}, J=6.8 \mathrm{~Hz}, 6 \mathrm{H}, \mathrm{Z}$-isomer), 1.35 (s, 3H, Z-isomer), 2.29 (s, 3H, Z-isomer), 2.33 (s, 3H, E-isomer), 3.18 (s, 2H, Z-isomer), 3.48 (s, 2H, E-isomer), 3.63-3.71 (m, 2H), 3.79-3,87 (m, 4H), 3.91-3.99 (m, 2H), 6.44 (s, 1H, Z-isomer), 6.69 (s, 1H, E-isomer), 
6.86-6.88 (m, 2H), 6.98-7.11 (m, 9H), 7.19-7.35 (m, 7H). ${ }^{13} \mathrm{C}$ NMR (100 MHz, CDCl 3$) \delta 13.8$ (E-isomer), 14.0 (Z-isomer), 19.6 ( $E$-isomer), 19.9 (Z-isomer), 21.2 (Z-isomer), 21.3 ( $E$-isomer), 34.0 ( $E$-isomer), 45.1 (Z-isomer), 53.3 (E-isomer), 53.6 (Z-isomer), 60.95 (E-isomer), 61.01 (Z-isomer), 126.2 (Z-isomer), 126.4 ( $(E$-isomer), 127.2 (E-isomer), 127.6 (Z-isomer), 128.1 (Z-isomer), 128.4 (E-isomer), 128.6 (E-isomer), 128.7 (Z-isomer), 128.8 (Z-isomer), 129.0 (E-isomer), 130.9 (Z-isomer), 132.9 ( $(E$-isomer), 136.5 (Z-isomer), 136.6 (E-isomer), 136.7 (E-isomer), 136.9 (Z-isomer), 137.5 (Z-isomer), 137.7 (E-isomer), 138.4 (Z-isomer), 140.0 (E-isomer), 171.47 ( $Z$-isomer), 171.49 ( $E$-isomer). HRMS (EI) $\mathrm{m} / \mathrm{z}$ calcd for $\mathrm{C}_{24} \mathrm{H}_{28} \mathrm{O}_{4}$ : 380.1988 , found 380.1992 .

Typical Procedure for Rh-Catalyzed Cyclization of 4 Bearing Diester Moiety (Scheme 5). To a mixture of $[\mathrm{RhCl}(\mathrm{cod})]_{2}(1.8 \mathrm{mg}, 3.68 \mu \mathrm{mol}, 2.5 \mathrm{~mol} \%)$, dppb (3.1 mg, $\left.7.4 \mu \mathrm{mol}, 5 \mathrm{~mol} \%\right)$, and $\mathrm{Cs}_{2} \mathrm{CO}_{3}$ (144.0 mg, $0.44 \mathrm{mmol}$ ) was added a solution of 4 aa $(63.3 \mathrm{mg}, 0.15 \mathrm{mmol})$ in dioxane $(1.5 \mathrm{~mL})$ and $\mathrm{H}_{2} \mathrm{O}$ $(8.0 \mathrm{mg}, 0.44 \mathrm{mmol})$ at room temperature. The resultant mixture was stirred at $90{ }^{\circ} \mathrm{C}$ for $5 \mathrm{~h}$. After cooling the reaction mixture to room temperature, the catalyst and salt were removed by filtration through a short silica gel pad (EtOAc). The filtrate was evaporated and the residue was subjected to silica gel chromatography (hexane/EtOAc $=6 / 1)$ to afford 7aa $(34.0 \mathrm{mg}, 89 \%)$ as colorless oil.

7aa: 89\% yield from 4aa. ${ }^{1} \mathrm{H}$ NMR $\left(400 \mathrm{MHz}, \mathrm{CDCl}_{3}\right) \delta 1.23(\mathrm{t}, J=6.8 \mathrm{~Hz}, 3 \mathrm{H}), 1.50(\mathrm{~s}, 3 \mathrm{H}), 2.42(\mathrm{~s}, 3 \mathrm{H})$, $2.87(\mathrm{dd}, J=18.0,1.6 \mathrm{~Hz}, 1 \mathrm{H}), 3.60(\mathrm{dd}, J=18.0,1.6 \mathrm{~Hz}, 1 \mathrm{H}), 4.12-4.20(\mathrm{~m}, 2 \mathrm{H}), 6.48(\mathrm{t}, J=1.6 \mathrm{~Hz}, 1 \mathrm{H})$, $7.26(\mathrm{~d}, J=8.0 \mathrm{~Hz}, 2 \mathrm{H}), 7.55(\mathrm{~d}, J=8.0 \mathrm{~Hz}, 2 \mathrm{H}) .{ }^{13} \mathrm{C} \mathrm{NMR}\left(100 \mathrm{MHz}, \mathrm{CDCl}_{3}\right) \delta 14.2,21.3,21.7,42.3,54.9$, 61.5, 123.4, 126.8, 129.5, 130.6, 142.2, 171.6, 171.7, 205.1. HRMS (EI) $\mathrm{m} / \mathrm{z}$ calcd for $\mathrm{C}_{16} \mathrm{H}_{18} \mathrm{O}_{3}: 258.1256$, found 258.1255 .

7ab: 88\% yield from 4 ab. ${ }^{1} \mathrm{H}$ NMR $\left(400 \mathrm{MHz}, \mathrm{CDCl}_{3}\right) \delta 1.23(\mathrm{t}, J=6.8 \mathrm{~Hz}, 3 \mathrm{H}), 1.50(\mathrm{~s}, 3 \mathrm{H}), 2.89(\mathrm{dd}, J=$ 18.0, $1.6 \mathrm{~Hz}, 1 \mathrm{H}), 3.63(\mathrm{dd}, J=18.0,1.6 \mathrm{~Hz}, 1 \mathrm{H}), 4.13-4.21(\mathrm{~m}, 2 \mathrm{H}), 6.53(\mathrm{t}, J=1.6 \mathrm{~Hz}, 1 \mathrm{H}), 7.44-7.50(\mathrm{~m}$, $3 \mathrm{H}), 7.65-7.67(\mathrm{~m}, 2 \mathrm{H}) .{ }^{13} \mathrm{C}$ NMR $\left(100 \mathrm{MHz}, \mathrm{CDCl}_{3}\right) \delta 14.2,21.3,42.3,55.0,61.6,124.3,126.8,128.8,131.5$, 133.3, 171.5, 171.7, 205.1. HRMS (EI) $m / z$ calcd for $\mathrm{C}_{15} \mathrm{H}_{16} \mathrm{O}_{3}: 244.1099$, found 244.1100.

Typical Procedure for Rh-Catalyzed Cyclization of 4 Bearing Ketoester Moiety (Scheme 6). To a mixture of $[\mathrm{RhCl}(\mathrm{cod})]_{2}(2.0 \mathrm{mg}, 4.1 \mu \mathrm{mol}, 2.5 \mathrm{~mol} \%)$, dppb (3.6 mg, $\left.8.3 \mu \mathrm{mol}, 5 \mathrm{~mol} \%\right)$, and $\mathrm{Cs}_{2} \mathrm{CO}_{3}$ (162.0 mg, $0.50 \mathrm{mmol})$ was added a solution of $4 \mathbf{b b}(64.0 \mathrm{mg}, 0.17 \mathrm{mmol})$ in dioxane $(1.5 \mathrm{~mL})$ and $\mathrm{H}_{2} \mathrm{O}$ $(40 \mu \mathrm{L})$ at room temperature. The resultant mixture was stirred at $90{ }^{\circ} \mathrm{C}$ for $5 \mathrm{~h}$. After cooling the reaction mixture to room temperature, the catalyst and salt were removed by filtration through a short silica gel pad (EtOAc). The filtrate was evaporated and the residue was subjected to silica gel chromatography (hexane/EtOAc $=5 / 1$ to $4 / 1$, containing $\left.1 \% \mathrm{Et}_{3} \mathrm{~N}\right)$ to afford $10(38.9 \mathrm{mg}, 90 \%)$ as colorless oil.

10: $90 \%$ yield from $4 \mathbf{b b}$. This compound was obtained as a mixture of diastereomers $(\mathrm{major} / \mathrm{minor}=$ 84/16). ${ }^{1} \mathrm{H}$ NMR $\left(400 \mathrm{MHz}, \mathrm{C}_{6} \mathrm{D}_{6}\right) \delta 0.96(\mathrm{t}, J=7.2 \mathrm{~Hz}, 3 \mathrm{H}$, major isomer), $1.01(\mathrm{t}, J=7.2 \mathrm{~Hz}, 3 \mathrm{H}$, minor isomer), $1.21(\mathrm{~s}, 3 \mathrm{H}$, minor isomer), $1.37(\mathrm{~s}, 3 \mathrm{H}$, major isomer), $1.43(\mathrm{~s}, 3 \mathrm{H}$, major isomer), $1.51(\mathrm{~s}, 3 \mathrm{H}$, minor isomer), $2.34(\mathrm{dd}, J=16.4,0.8 \mathrm{~Hz}, 1 \mathrm{H}$, minor isomer), $2.46(\mathrm{dd}, J=16.0,0.8 \mathrm{~Hz}, 1 \mathrm{H}$, major isomer), $3.52(\mathrm{dd}, J=16.0,2.4 \mathrm{~Hz}, 1 \mathrm{H}$, major isomer), $3.82(\mathrm{dd}, J=16.4,2.4 \mathrm{~Hz}, 1 \mathrm{H}$, minor isomer), 3.92-4.02 (m, $2 \mathrm{H}$, major isomer), 4.01-4.10 (m, $2 \mathrm{H}$, minor isomer), $5.81(\mathrm{dd}, J=2.4,0.8 \mathrm{~Hz}, 1 \mathrm{H}$, minor isomer), 5.87 (dd, $J=2.4,0.8 \mathrm{~Hz}, 1 \mathrm{H}$, major isomer), 7.04-7.13 (m, 6H), 7.24-7.28 (m, 4H). ${ }^{13} \mathrm{C}$ NMR $\left(100 \mathrm{MHz}, \mathrm{C}_{6} \mathrm{D}_{6}\right) \delta 14.5$, 21.7 (major isomer), 22.7 (minor isomer), 23.1 (minor isomer), 25.3 (major isomer), 42.7 (major isomer), 43.3 (minor isomer), 56.4 (minor isomer), 57.2 (major isomer), 60.5 (major isomer), 60.7 (minor isomer), 84.6 (major isomer), 85.3 (minor isomer), 126.1 (major isomer), 126.3 (minor isomer), 128.0 (major isomer), 128.2 (minor isomer), 128.3 (minor isomer), 128.6 (major isomer), 130.0 (minor isomer), 130.5 (major isomer), 135.9 (minor isomer), 136.0 (major isomer), 139.9 (major isomer), 142.8 (minor isomer), 174.9 (minor isomer), 175.4 (major isomer). HRMS (EI) $m / z$ calcd for $\mathrm{C}_{16} \mathrm{H}_{20} \mathrm{O}_{3}$ : 260.1412, found 260.1412. 
11: 93\% yield from 4cd. ${ }^{1} \mathrm{H}$ NMR $\left(400 \mathrm{MHz}, \mathrm{C}_{6} \mathrm{D}_{6}\right) \delta 0.99(\mathrm{t}, J=7.2 \mathrm{~Hz}, 3 \mathrm{H}), 1.10-1.31(\mathrm{~m}, 3 \mathrm{H}), 1.59-1.71$ $(\mathrm{m}, 2 \mathrm{H}), 1.93-2.02(\mathrm{~m}, 2 \mathrm{H}), 2.09-2.16(\mathrm{~m}, 1 \mathrm{H}), 2.31(\mathrm{~d}, J=16.0 \mathrm{~Hz}, 1 \mathrm{H}), 2.55(\mathrm{br}, 1 \mathrm{H}), 3.54(\mathrm{dd}, J=16.0,2.0$ $\mathrm{Hz}, 1 \mathrm{H}), 3.97-4.04(\mathrm{~m}, 2 \mathrm{H}), 5.67(\mathrm{~d}, J=2.0 \mathrm{~Hz}, 1 \mathrm{H}), 6.77(\mathrm{dd}, J=2.8,1.2 \mathrm{~Hz}, 1 \mathrm{H}), 6.83(\mathrm{dd}, J=4.8,2.8 \mathrm{~Hz}$, 1H), $7.01(\mathrm{dd}, J=4.8,1.2 \mathrm{~Hz}, 1 \mathrm{H}) .{ }^{13} \mathrm{C}$ NMR $\left(100 \mathrm{MHz}, \mathrm{C}_{6} \mathrm{D}_{6}\right) \delta 14.6,22.6,23.5,35.1,36.0,43.7,55.9,60.6$, 83.9, 122.1, 125.9, 126.0, 129.5, 138.6, 140.1, 175.0. HRMS (EI) $\mathrm{m} / z$ calcd for $\mathrm{C}_{16} \mathrm{H}_{20} \mathrm{O}_{3} \mathrm{~S}: 292.1133$, found 292.1133.

\section{References Cited in Supporting Information}

(1) (a) Crebbé, P.; Fillion, H.; André, D.; Luche, J.-L. J. Chem. Soc., Chem. Commun. 1979, 859. (b) Searles, S.; Li, Y.; Nassim, B.; Lopes, M.-T. R.; Tran, P. T.; Crabbé, P. J. Chem. Soc., Perkin Trans. 1 1984, 747. (c) Crabbé, P.; Nassim, B.; Lopes, M.-T. R. Organic Synthesis; Wiley: New York, 1990; Collect. Vol. VII, p 276.

(2) (a) Zheng, B.; Myers, A. G. J. Am. Chem. Soc. 1996, 118, 4492. For the preparation of o-nitrobenzenesulfonylhydrazine (NBSH), see: (b) Myers, A. G.; Zheng, B.; Movassaghi, M. J. Org. Chem. 1997, 62, 7507.

(3) Tonogaki, K.; Itami, K.; Yoshida, J. J. Am. Chem. Soc. 2006, 128, 1464. 
${ }^{1} \mathrm{H}$ NMR $\left(400 \mathrm{MHz}, \mathrm{CDCl}_{3}\right)$

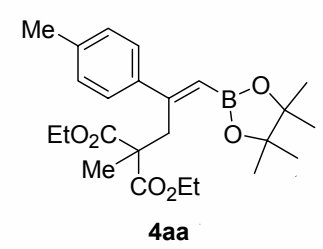

$\cos \cdot \mathrm{t}$

$280^{\circ} \mathrm{I}$

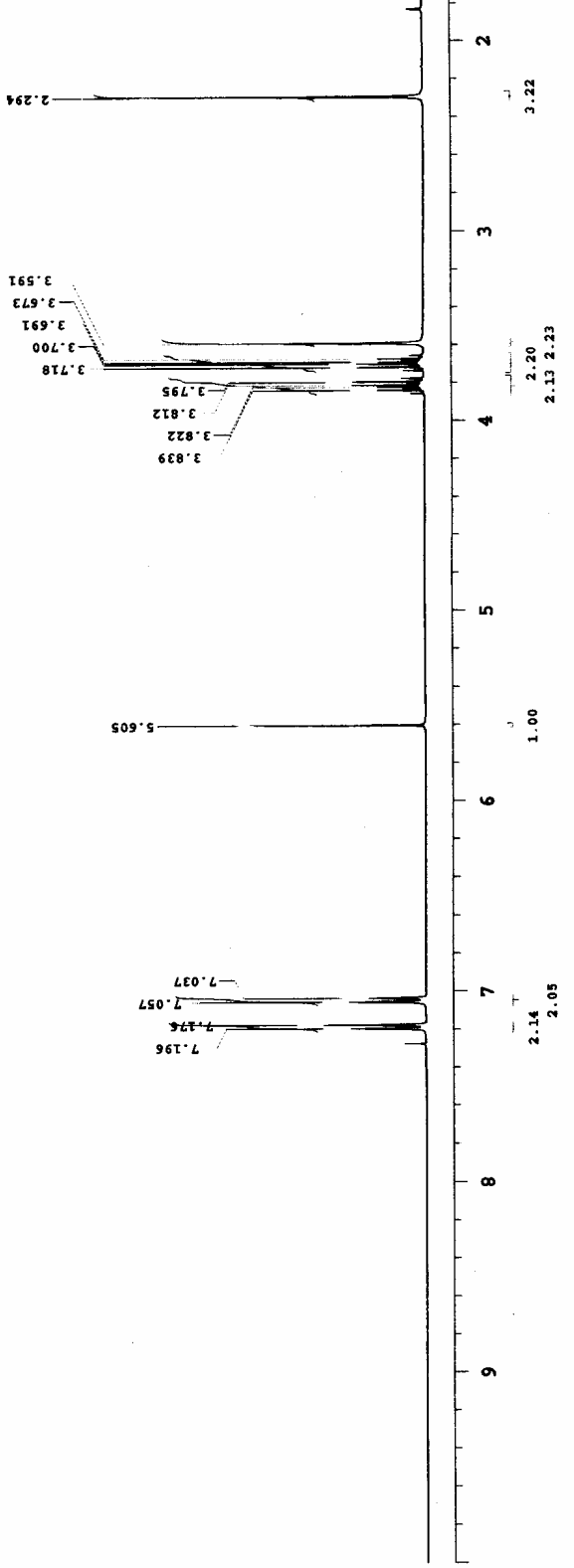


${ }^{13} \mathrm{C} \mathrm{NMR}\left(100 \mathrm{MHz}, \mathrm{CDCl}_{3}\right)$
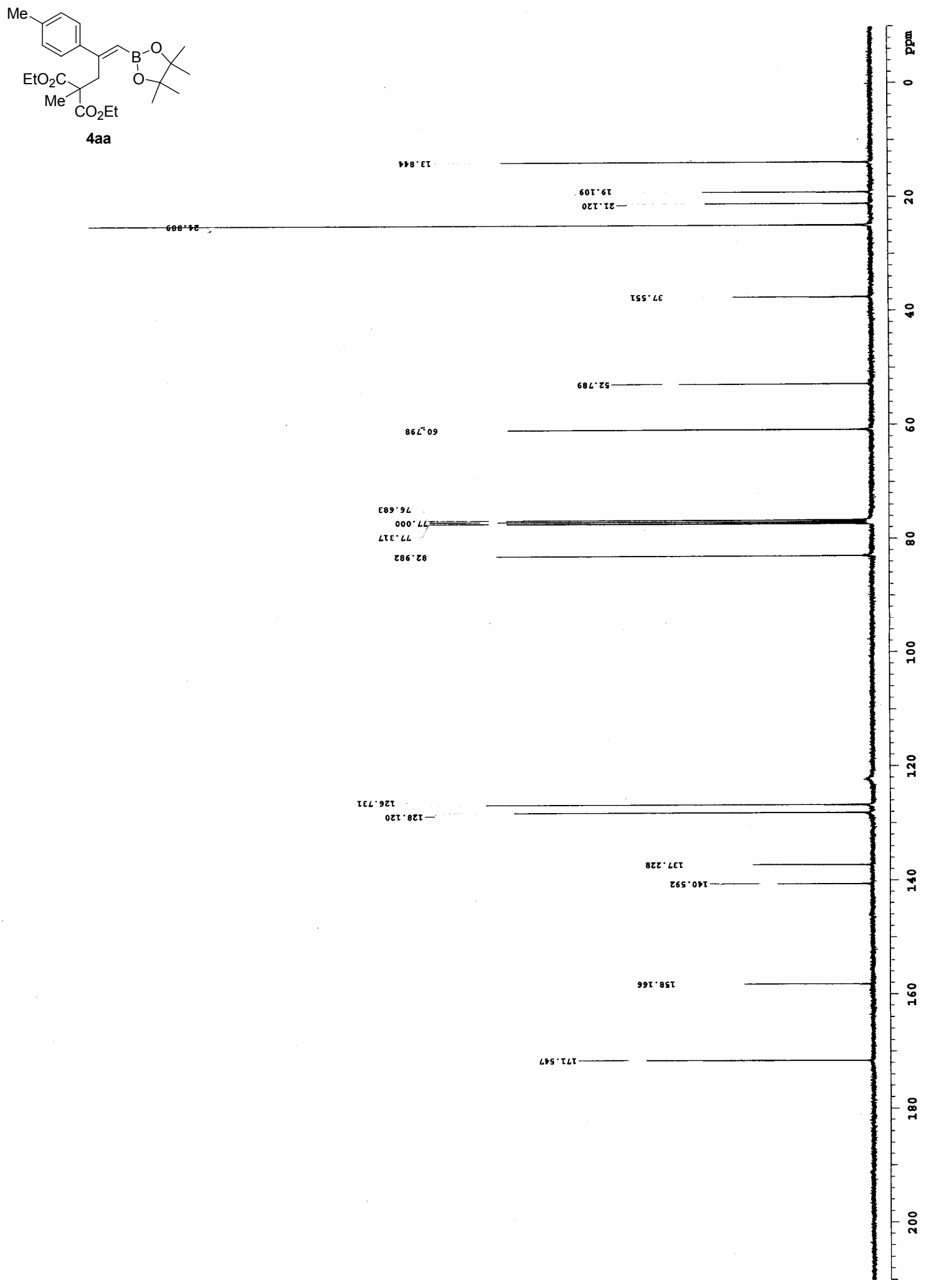
NOESY $\left(400 \mathrm{MHz}, \mathrm{CDCl}_{3}\right)$
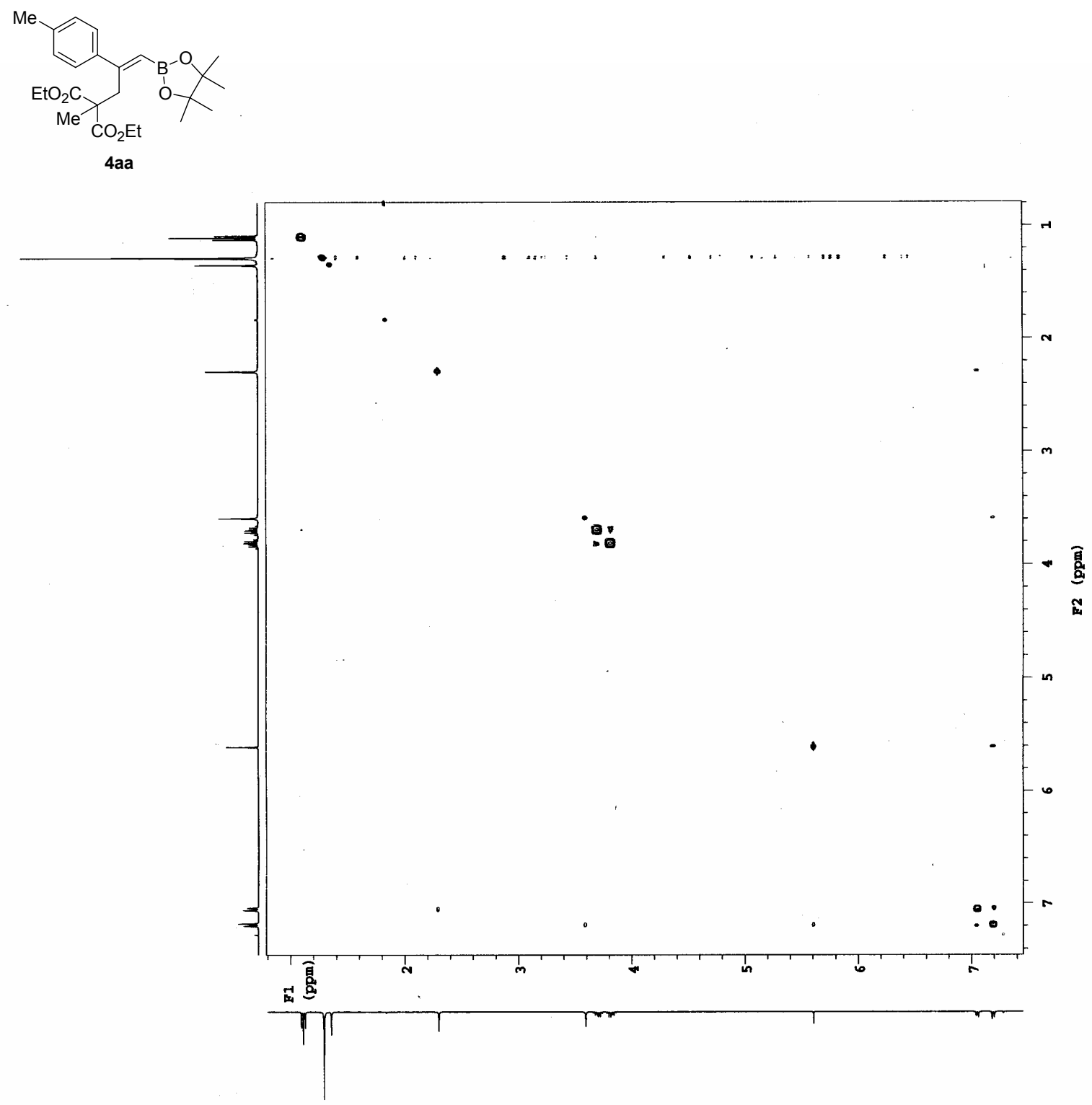
${ }^{1} \mathrm{H}$ NMR $\left(400 \mathrm{MHz}, \mathrm{CDCl}_{3}\right)$
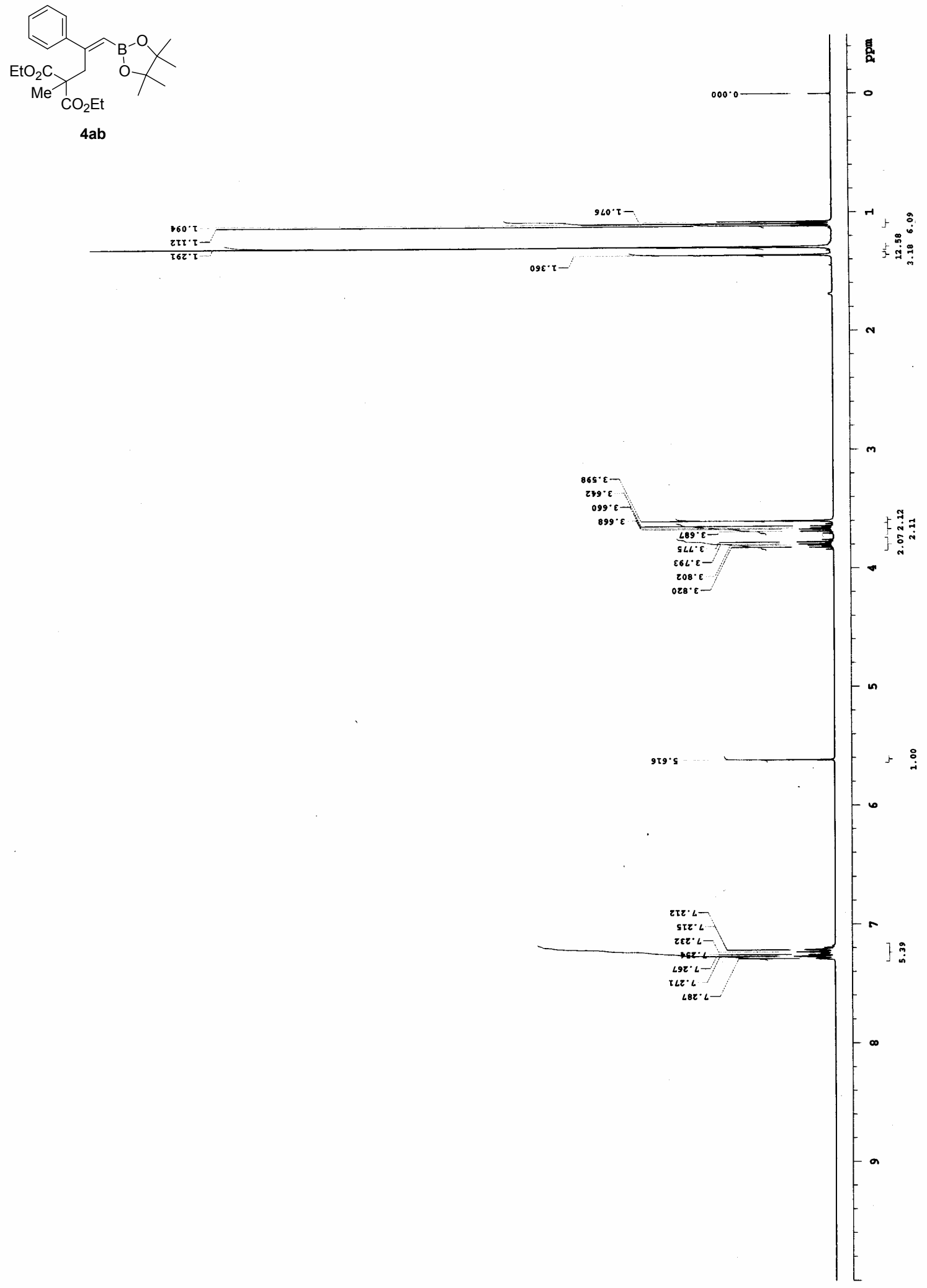
${ }^{13} \mathrm{C}$ NMR (100MHz, $\mathrm{CDCl}_{3}$ )
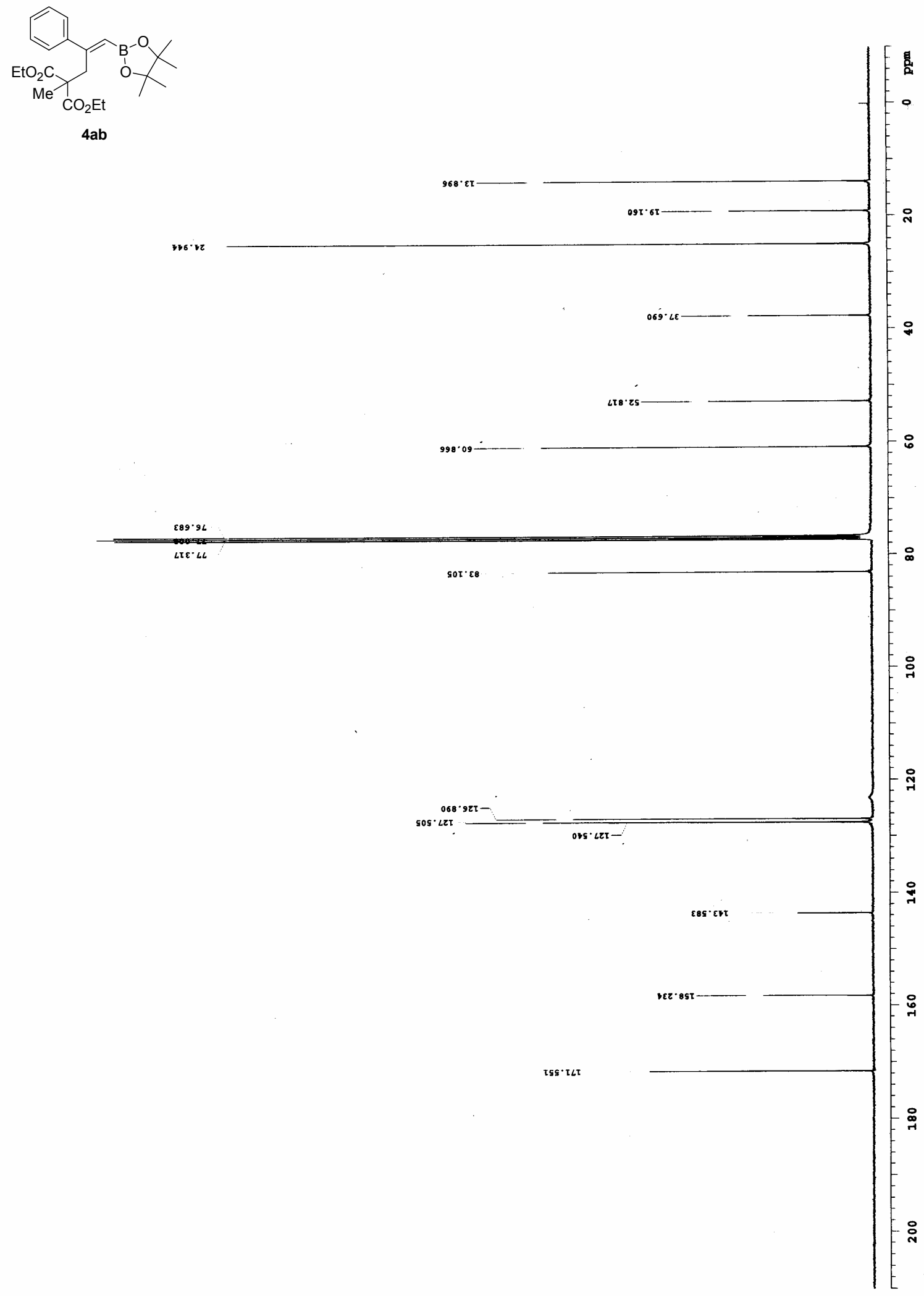
${ }^{1} \mathrm{H}$ NMR $\left(400 \mathrm{MHz}, \mathrm{CDCl}_{3}\right)$
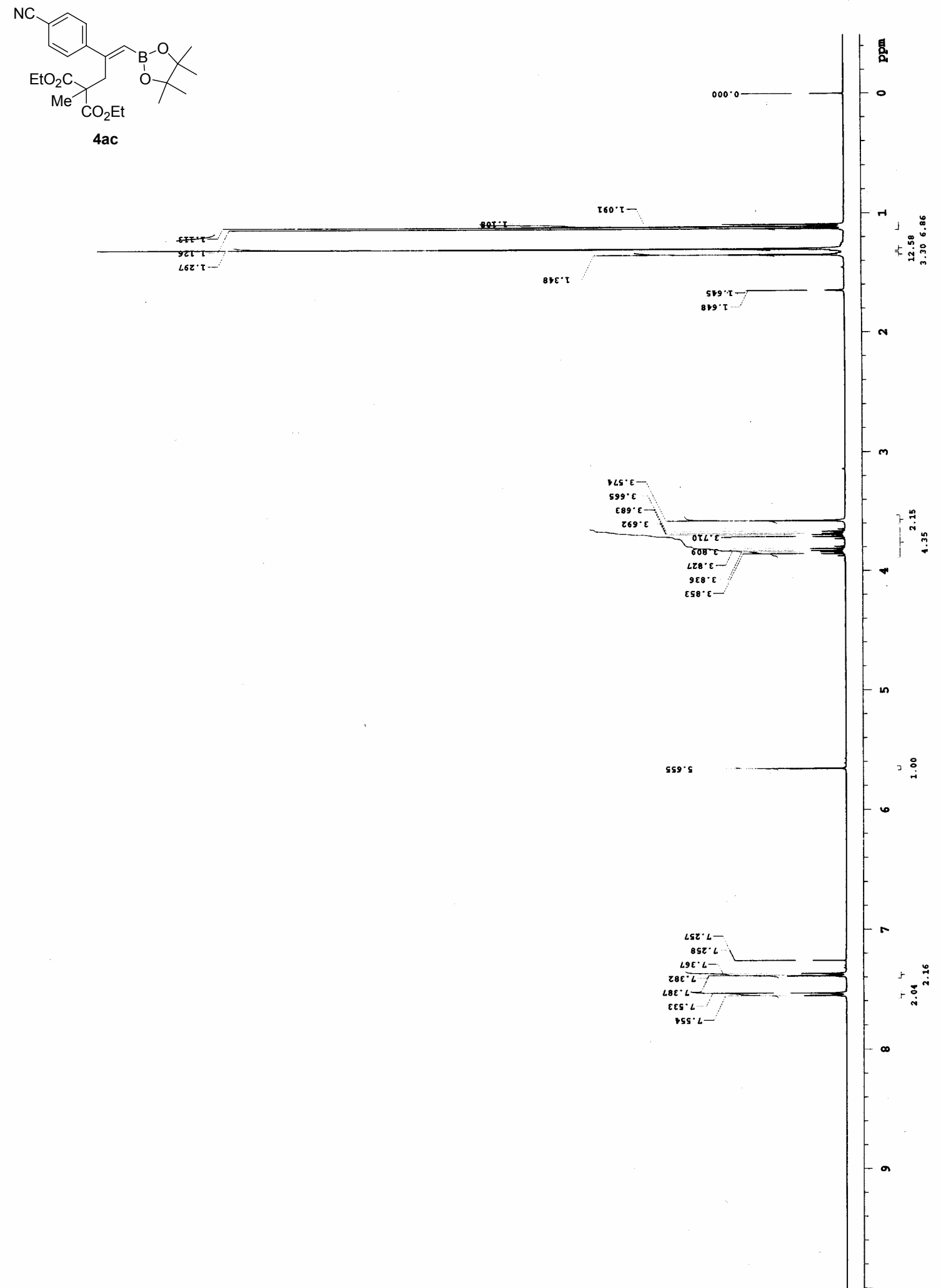
${ }^{13} \mathrm{C} \mathrm{NMR}\left(100 \mathrm{MHz}, \mathrm{CDCl}_{3}\right)$
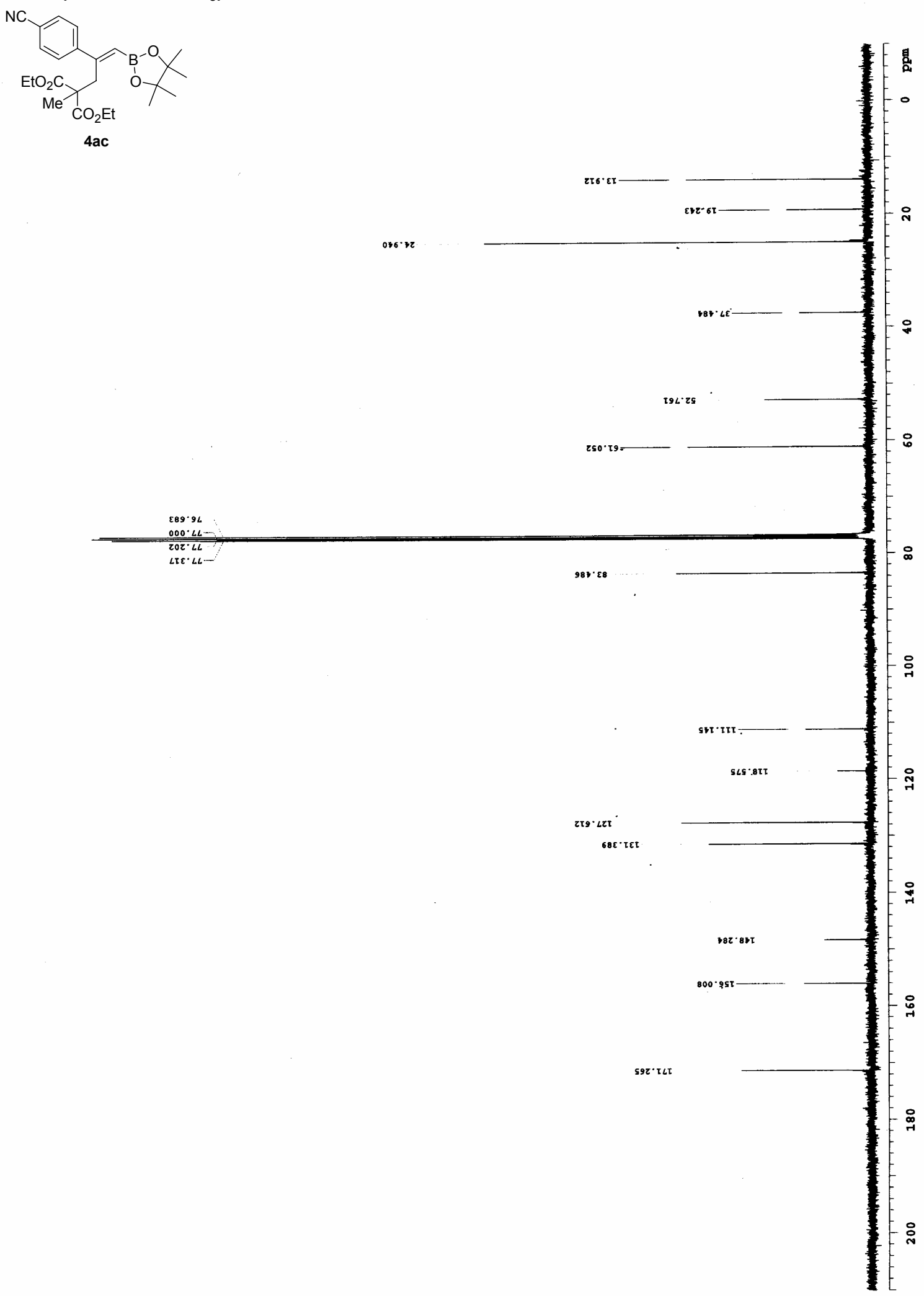

S11 
${ }^{1} \mathrm{H}$ NMR $\left(400 \mathrm{MHz}, \mathrm{CDCl}_{3}\right)$
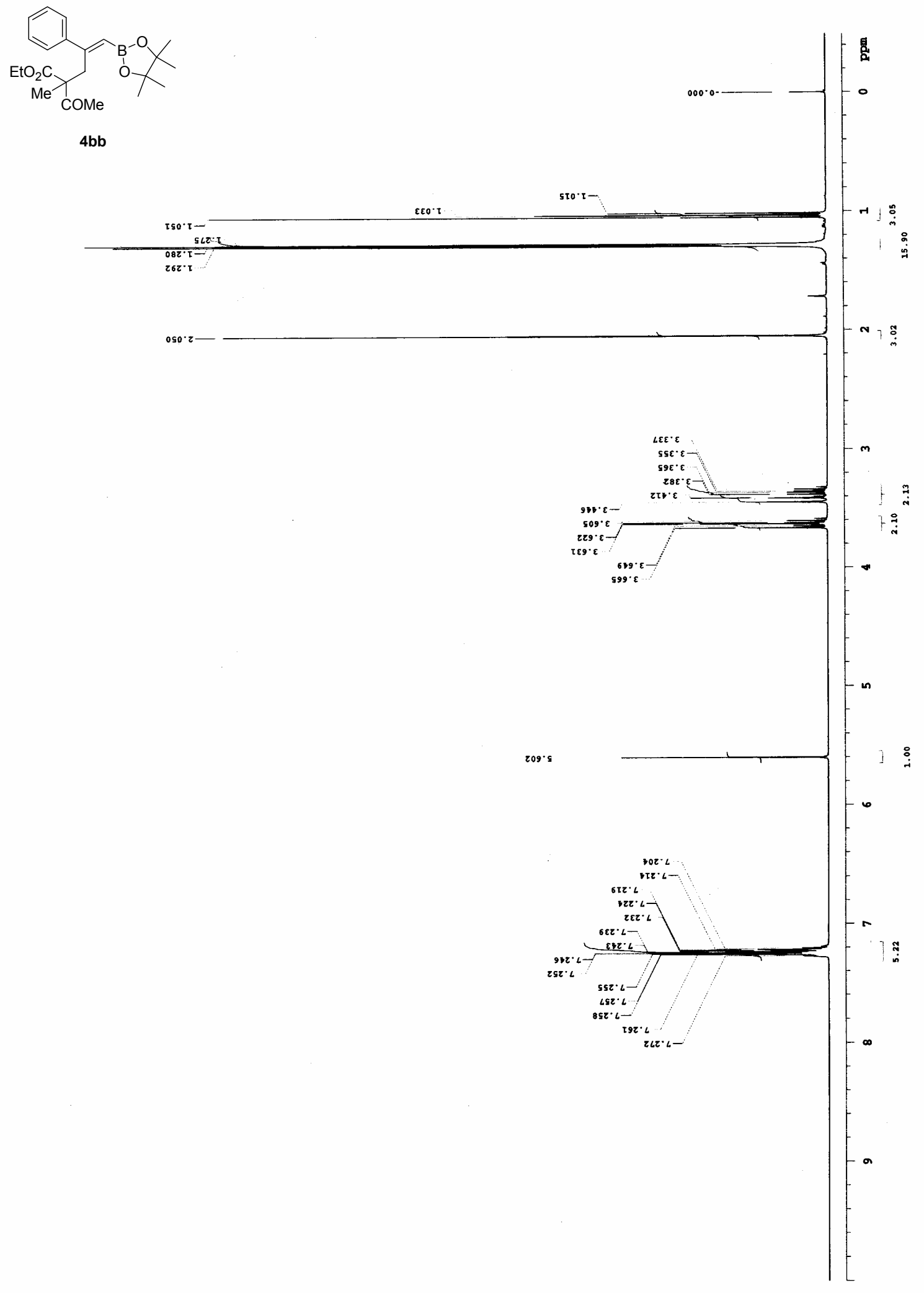
${ }^{13} \mathrm{C} \mathrm{NMR}\left(100 \mathrm{MHz}, \mathrm{CDCl}_{3}\right)$
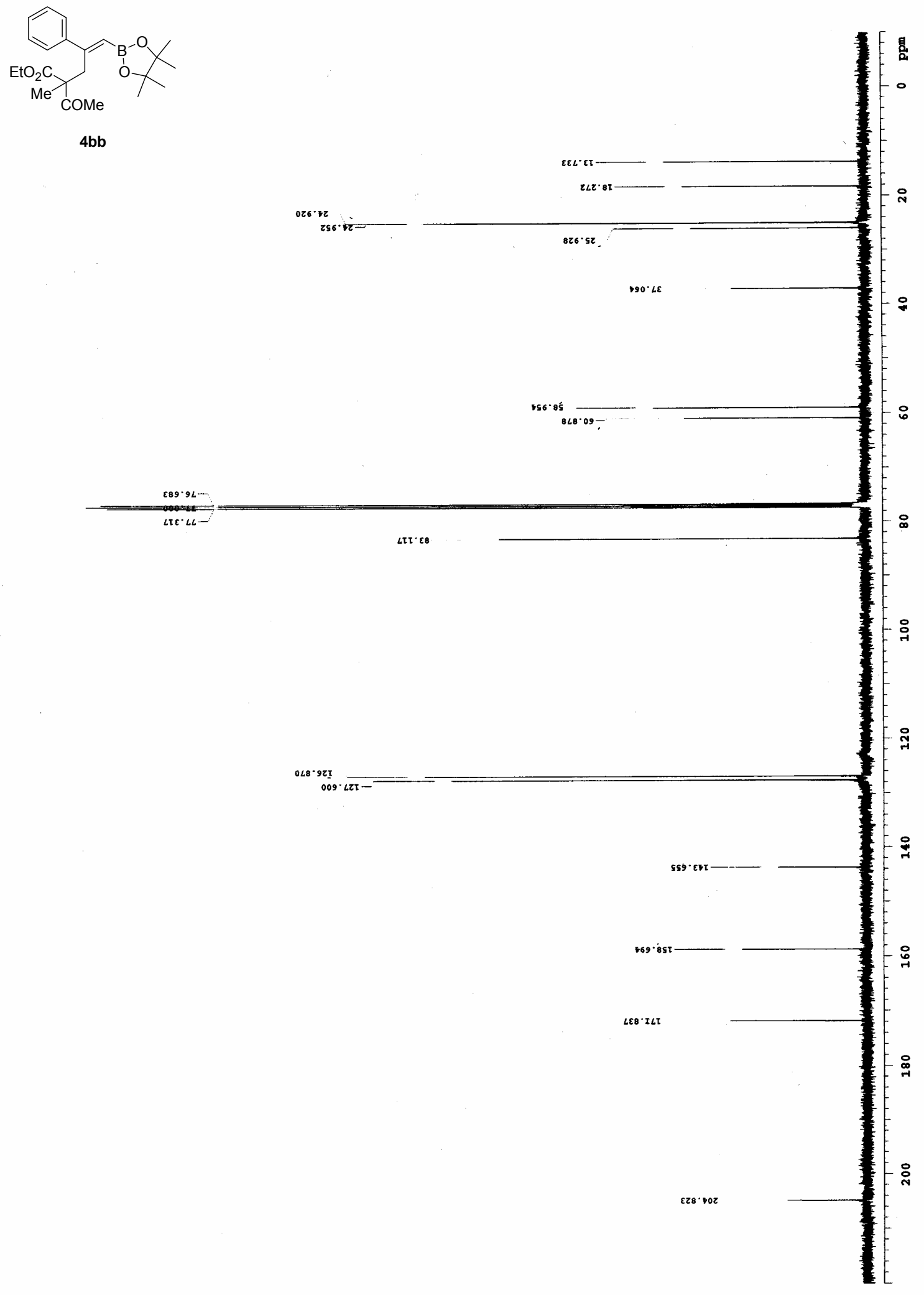
${ }^{1} \mathrm{H}$ NMR $\left(400 \mathrm{MHz}, \mathrm{CDCl}_{3}\right)$

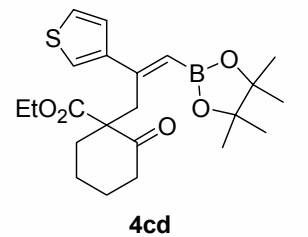

4cd

$292 \cdot x-1$
$0<z \cdot t-2$

$0 L Z \cdot \tau-$
$\varepsilon L S \cdot I$

เ65. 5

sta.t

0 9. 5

T89.

โ69. $\mathrm{T}$

$569^{\circ} \cdot \mathrm{T}$
$\mathrm{L} 66^{\circ} \mathrm{T}$

$466^{\circ} \mathrm{I}$
$\angle 6 \tau^{\circ} \mathrm{z}$

zนร. $z$

SIE. $z$

$z \varepsilon z \cdot z$

$6 \mathrm{Sb}$

จงย $\varepsilon-$

$28 \mathrm{E}$

OEV $\cdot \varepsilon-$
S9V- $\varepsilon$

$59 \mathrm{r} \cdot \varepsilon$
$\mathrm{szs} \cdot \varepsilon-$

เeต' $\varepsilon$
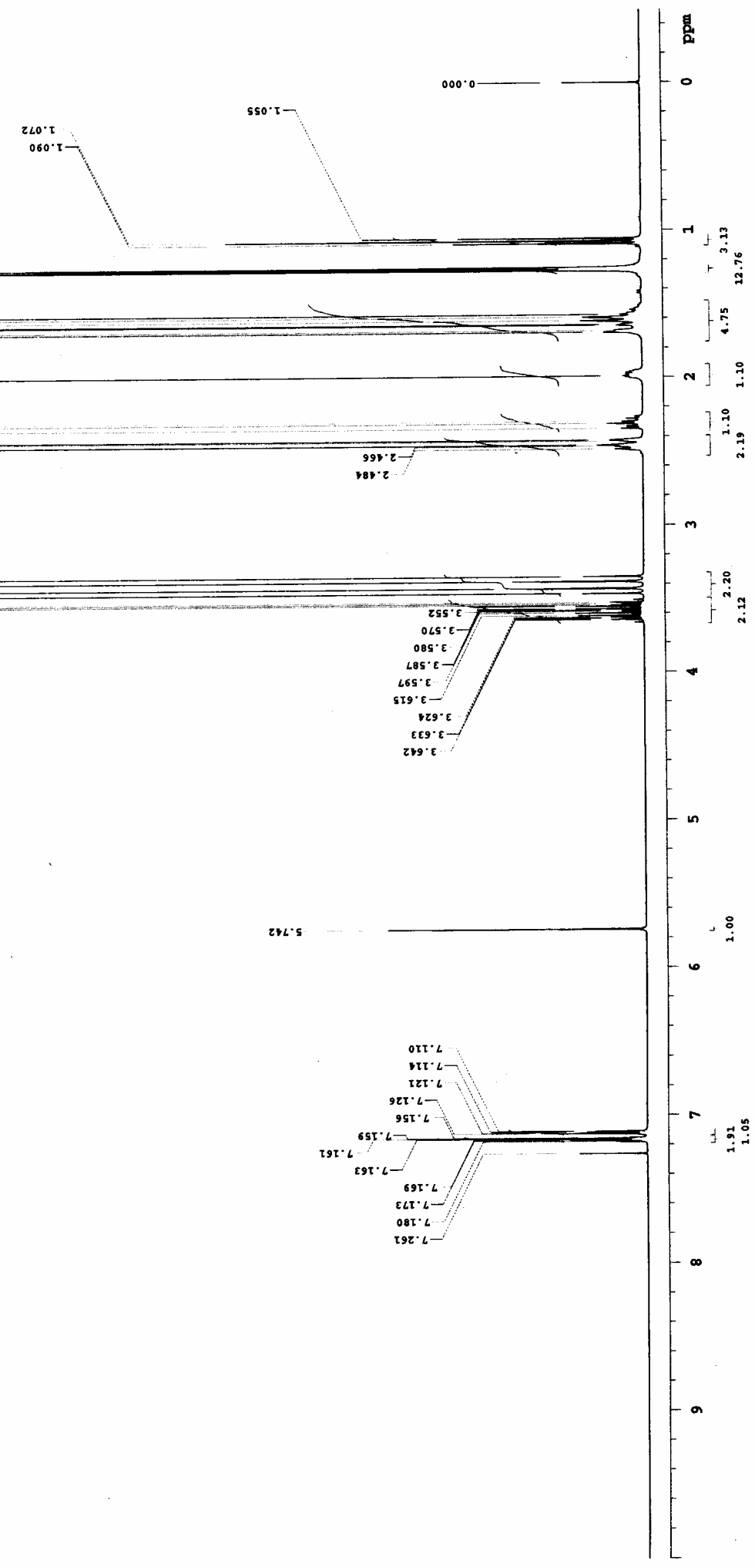
${ }^{13} \mathrm{C} \mathrm{NMR}\left(100 \mathrm{MHz}, \mathrm{CDCl}_{3}\right)$
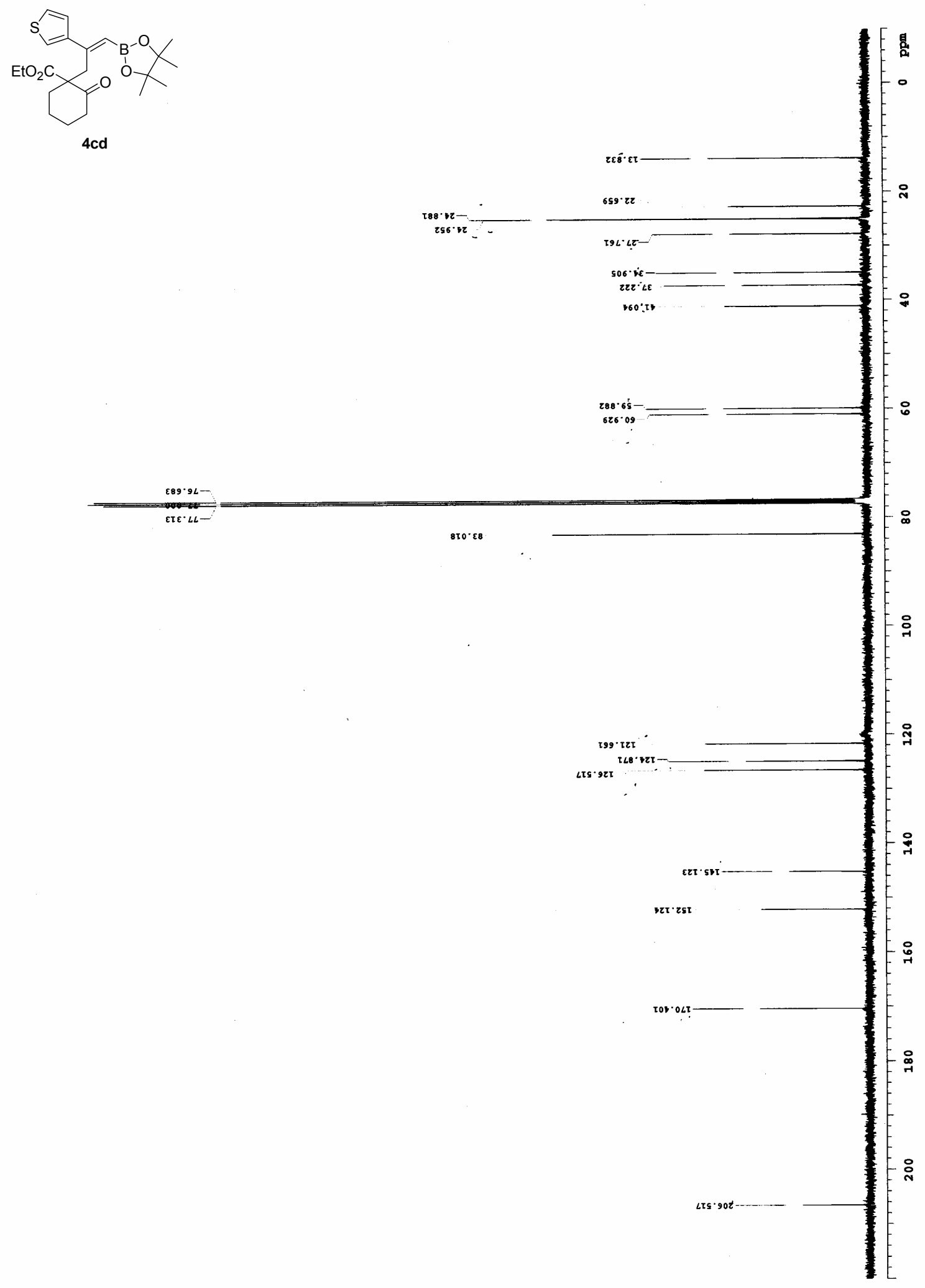
${ }^{1} \mathrm{H}$ NMR $\left(400 \mathrm{MHz}, \mathrm{CDCl}_{3}\right)$
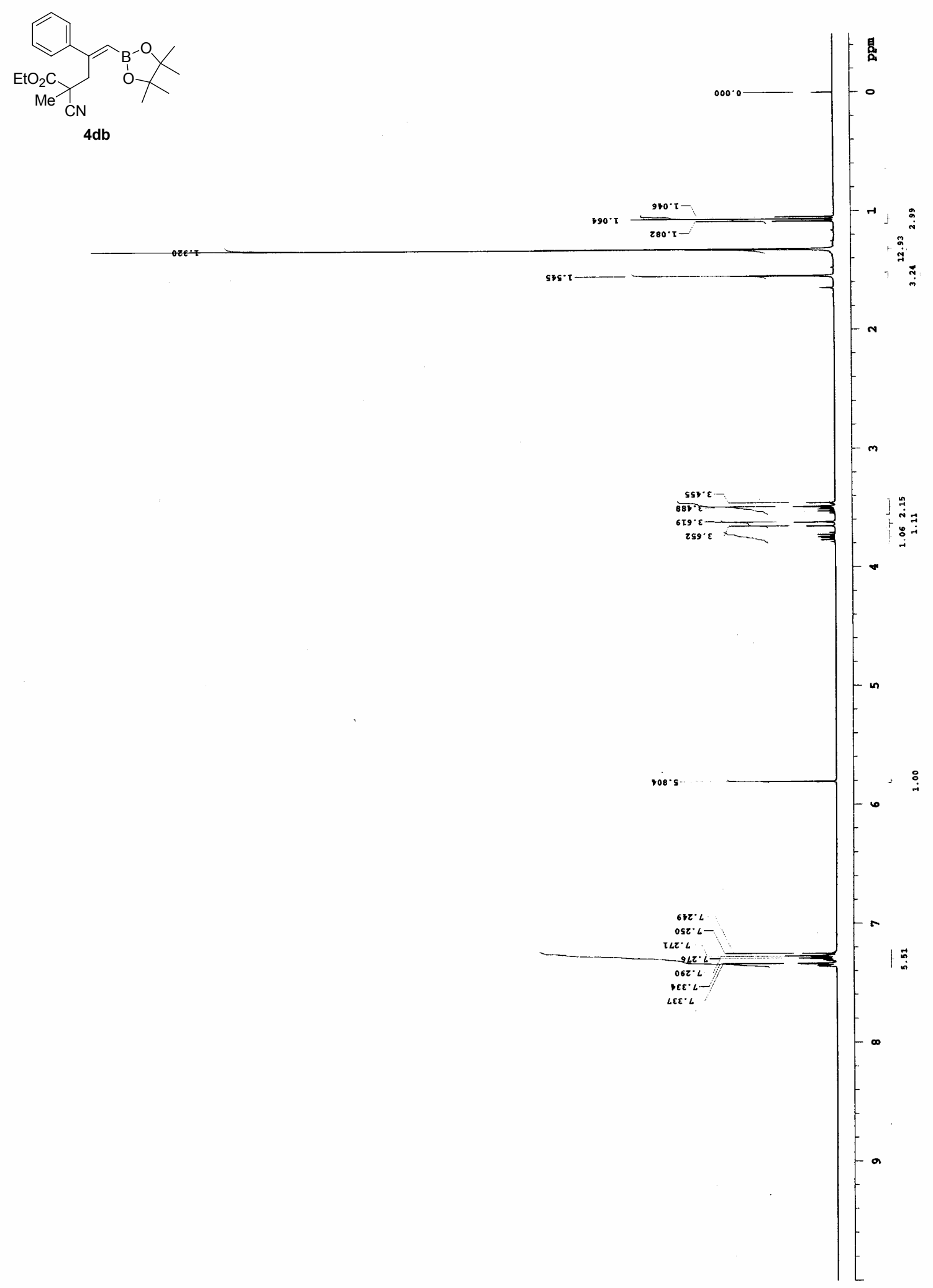
${ }^{13} \mathrm{C} \mathrm{NMR}\left(100 \mathrm{MHz}, \mathrm{CDCl}_{3}\right)$
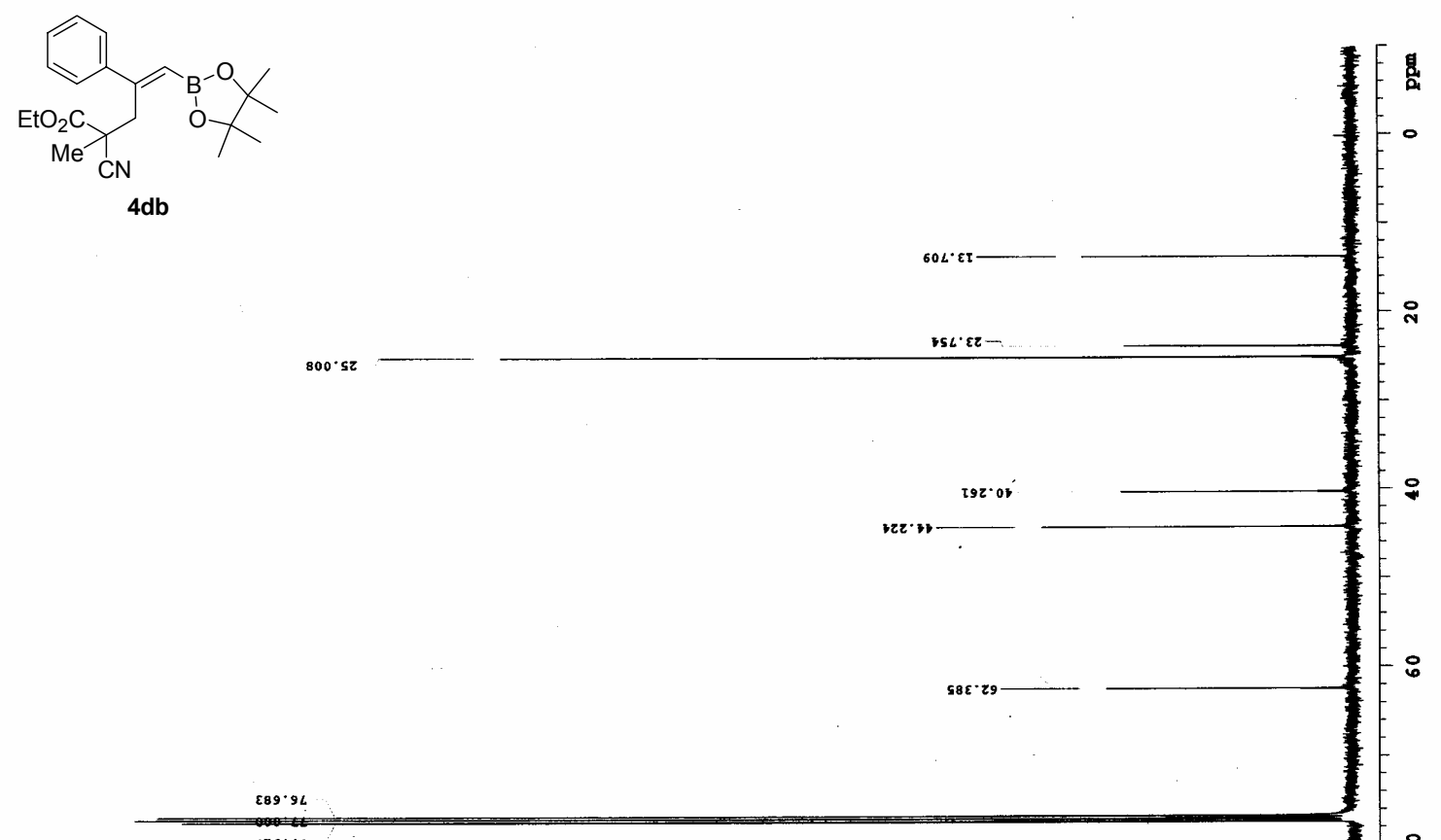

เIE. $\angle L$

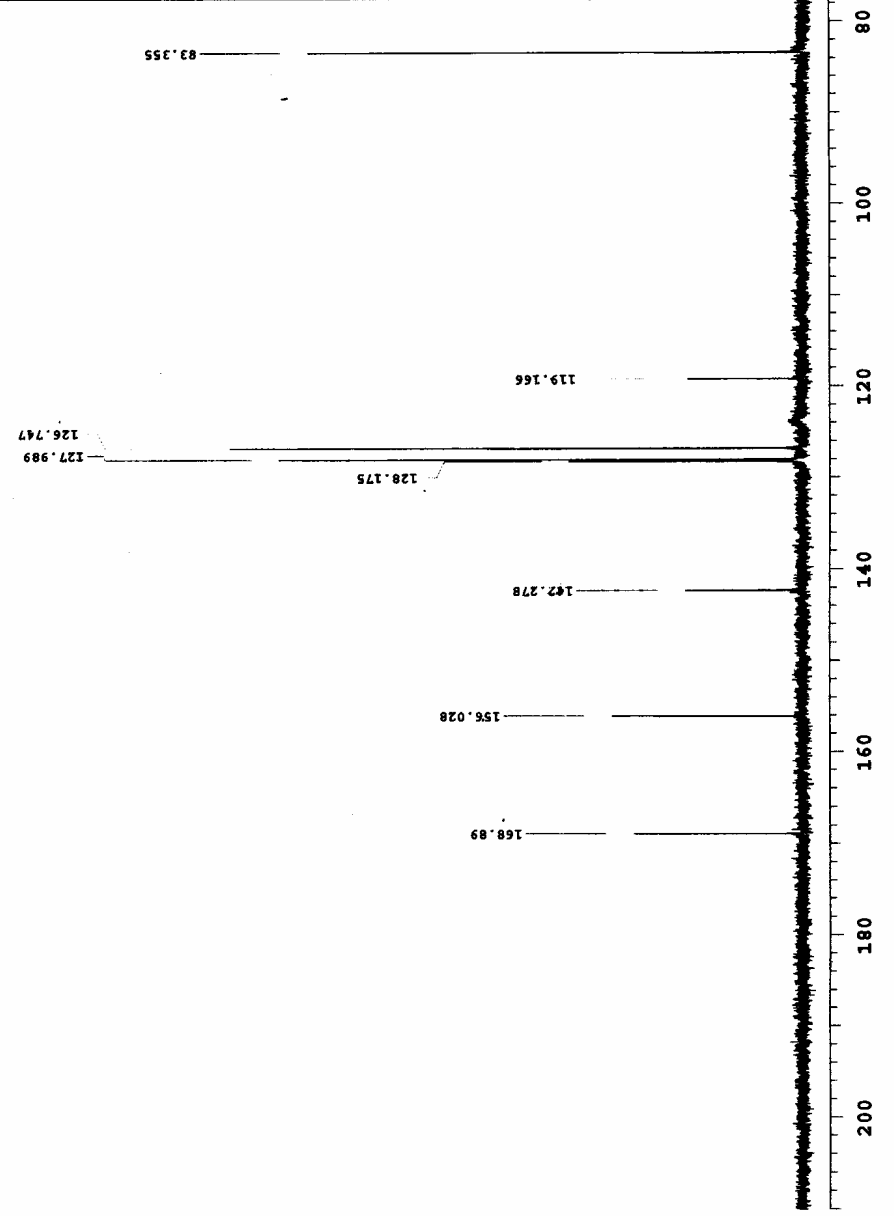


${ }^{1} \mathrm{H}$ NMR $\left(400 \mathrm{MHz}, \mathrm{CDCl}_{3}\right)$
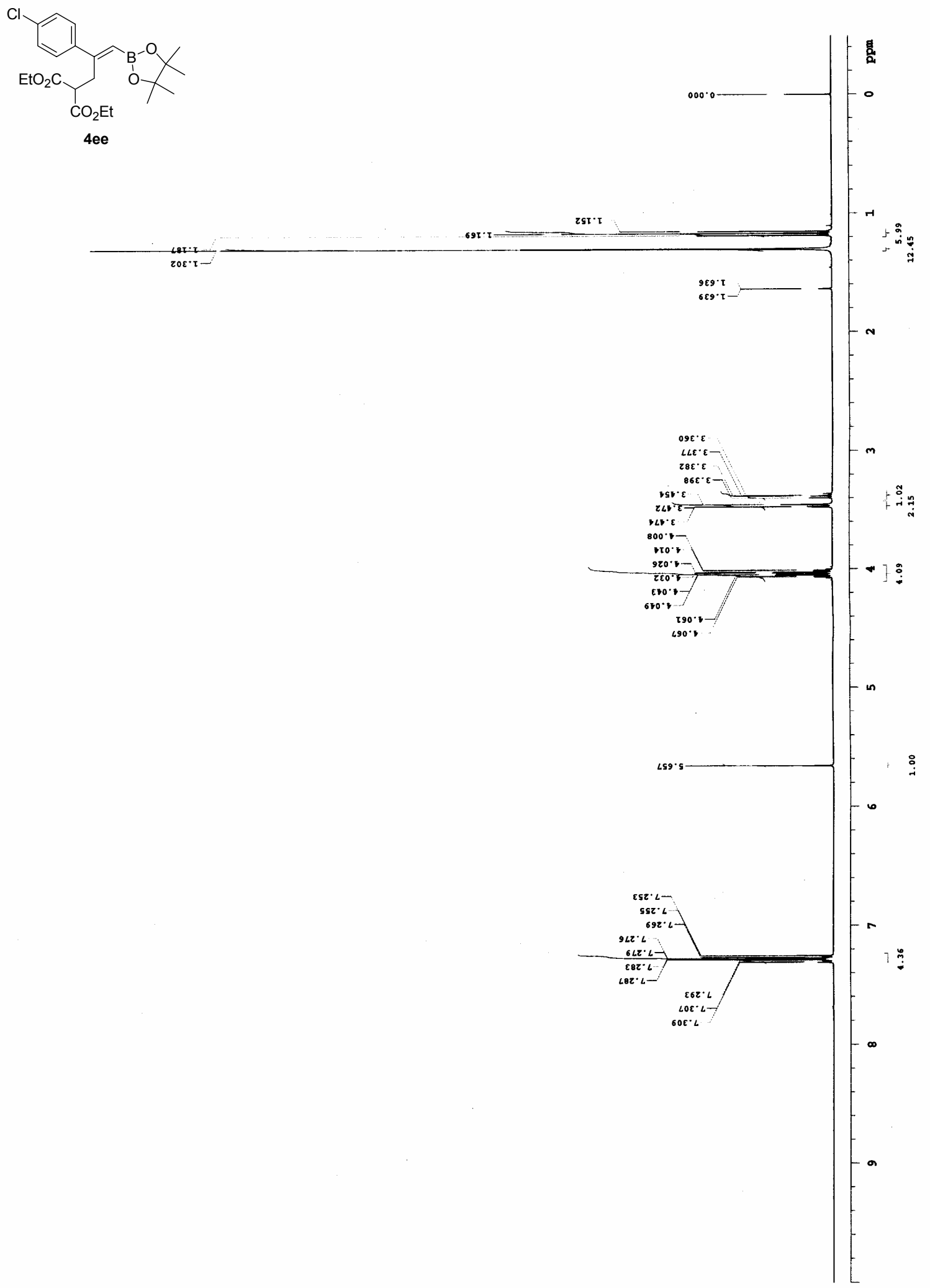
${ }^{13} \mathrm{C} \mathrm{NMR}\left(100 \mathrm{MHz}, \mathrm{CDCl}_{3}\right)$
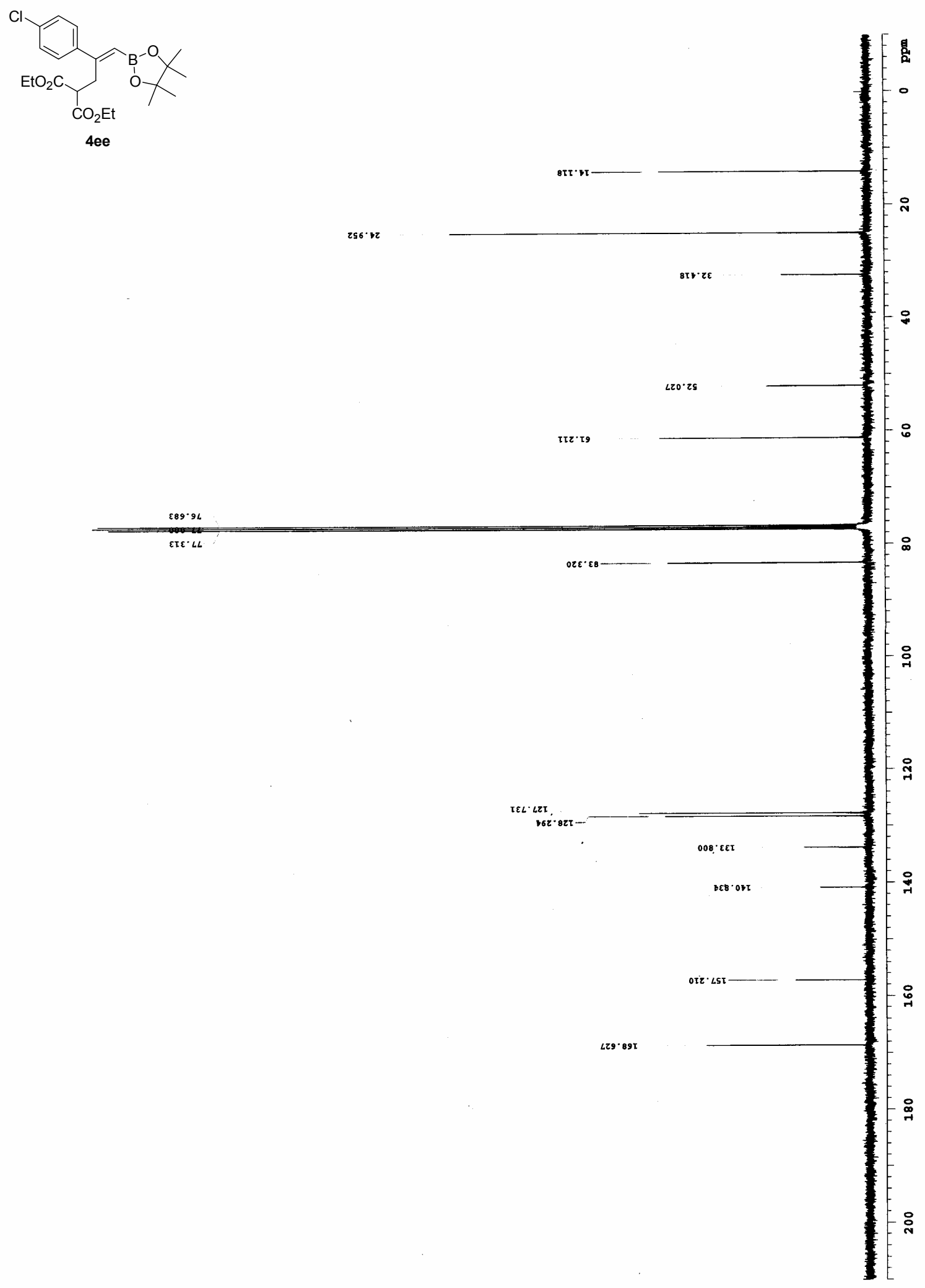
${ }^{1} \mathrm{H}$ NMR $\left(400 \mathrm{MHz}, \mathrm{CDCl}_{3}\right)$

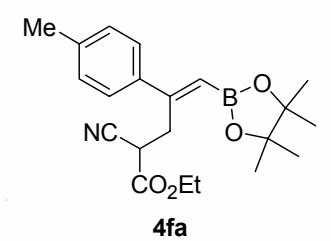

$4 \mathrm{fa}$

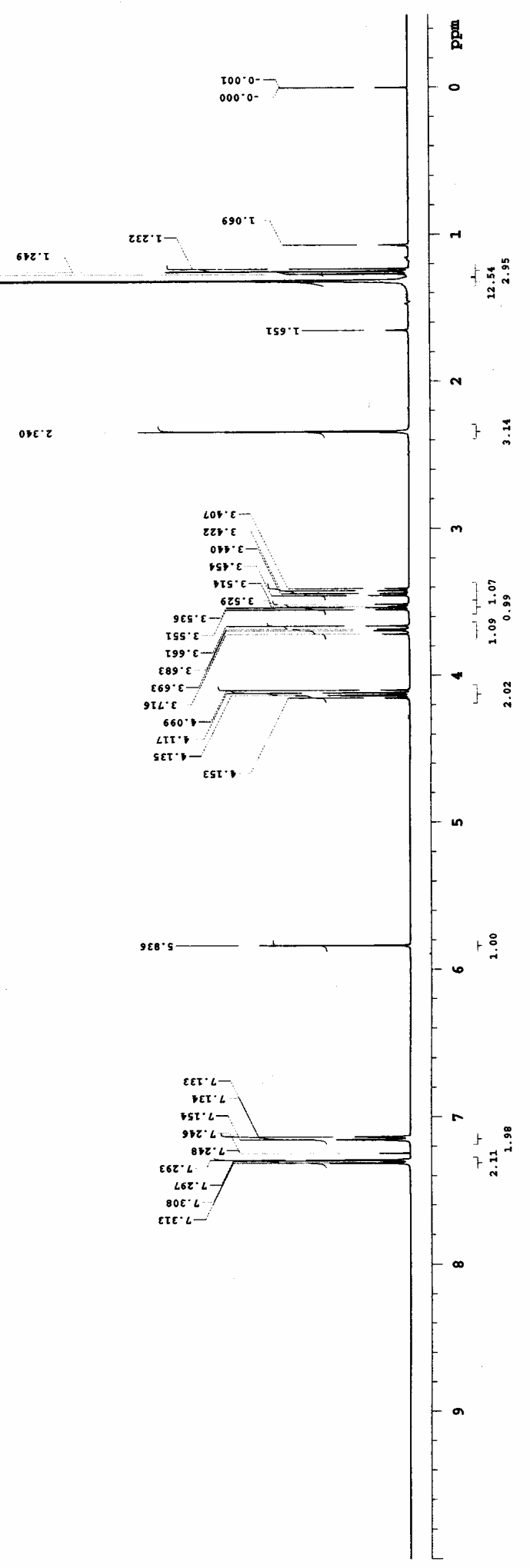


${ }^{13} \mathrm{C} \mathrm{NMR}\left(100 \mathrm{MHz}, \mathrm{CDCl}_{3}\right)$
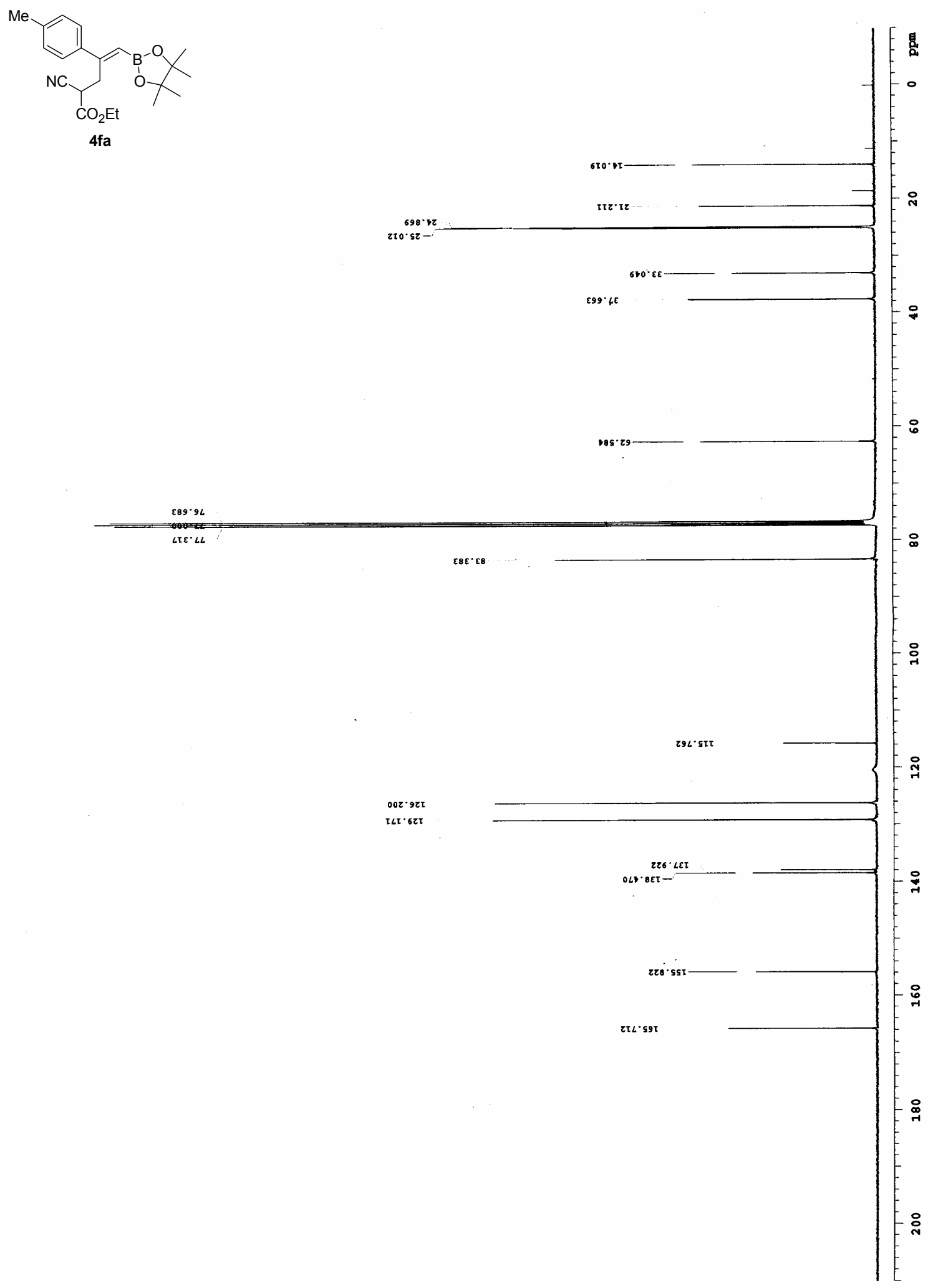
${ }^{1} \mathrm{H}$ NMR (400MHz, $\mathrm{CDCl}_{3}$ )<smiles>CCOC(=O)OCC</smiles>

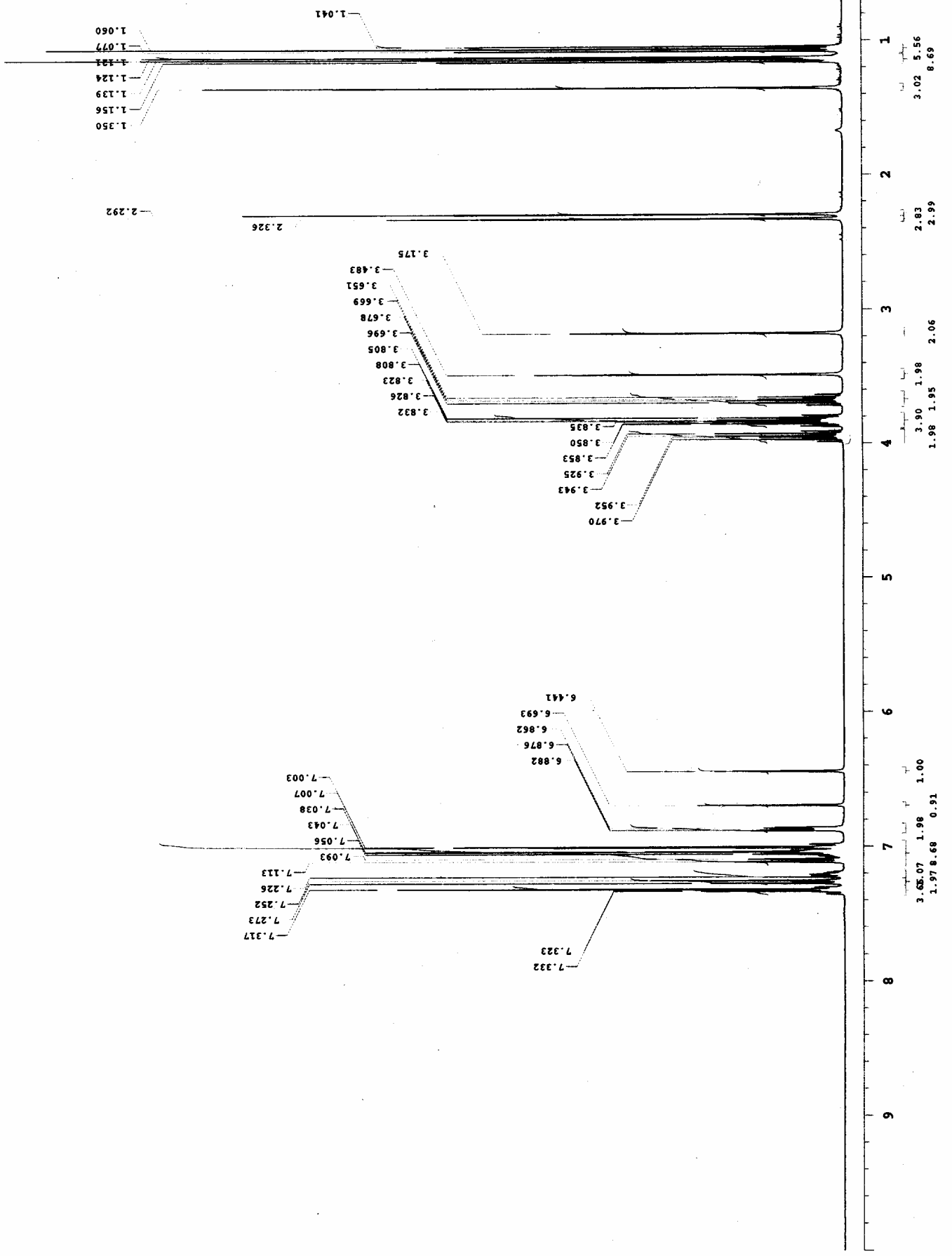


${ }^{13} \mathrm{C}$ NMR (100MHz, $\mathrm{CDCl}_{3}$ )
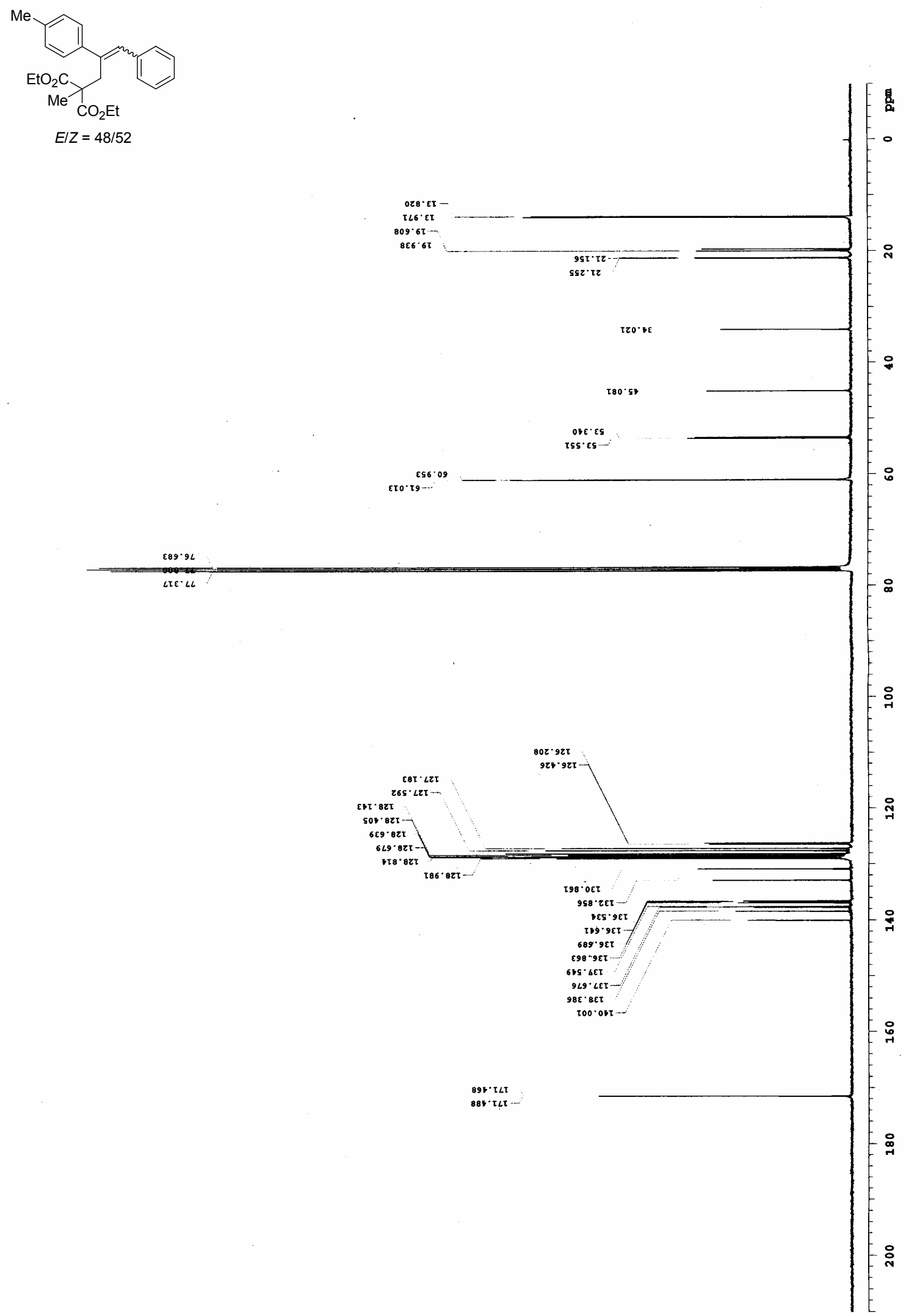
${ }^{1} \mathrm{H}$ NMR (400MHz, $\left.\mathrm{CDCl}_{3}\right)$
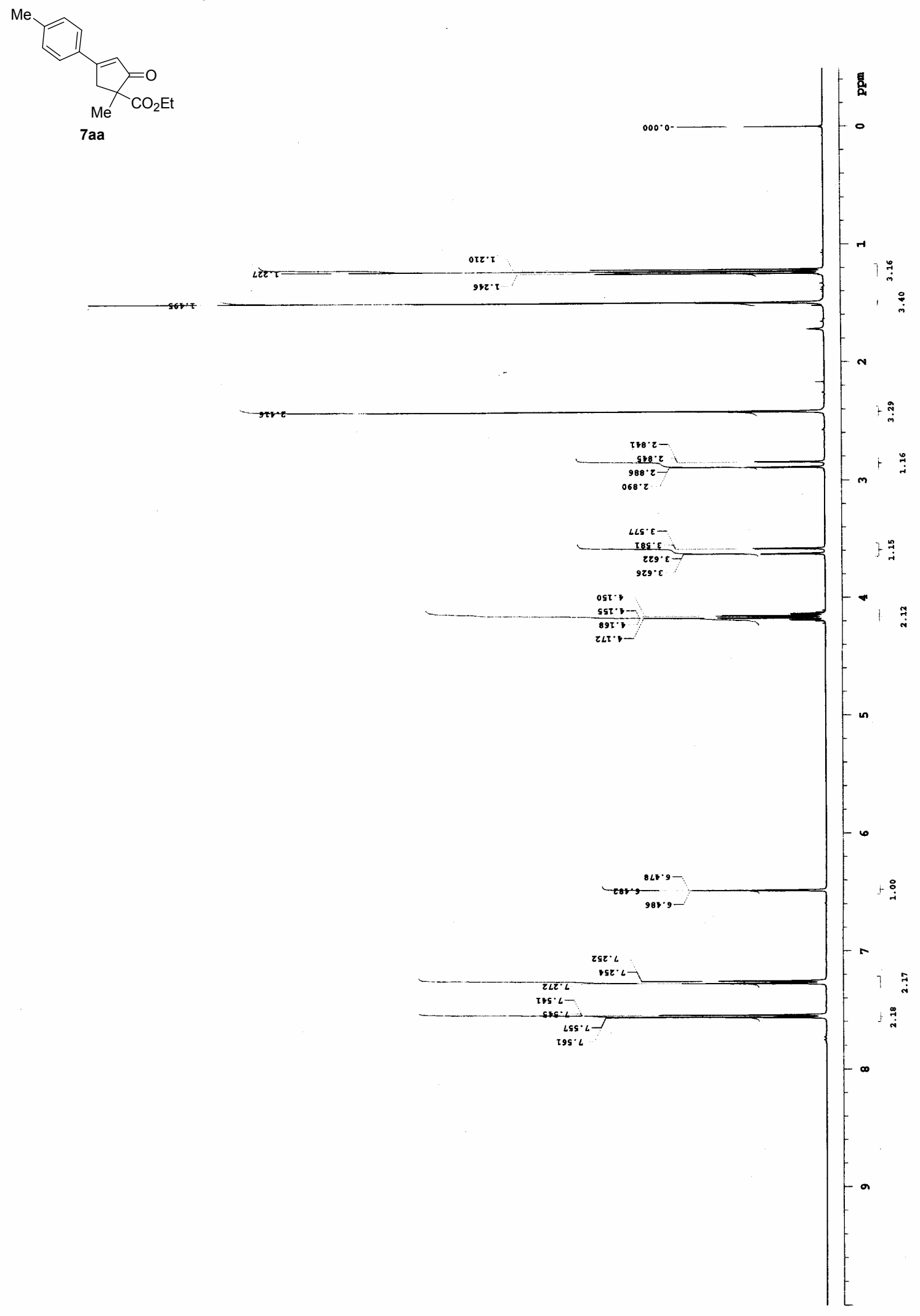
${ }^{13} \mathrm{C} \mathrm{NMR}\left(100 \mathrm{MHz}, \mathrm{CDCl}_{3}\right)$

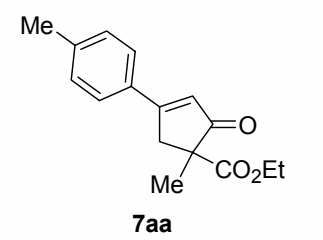

$889 \cdot 94-$

โโE $\angle L-$

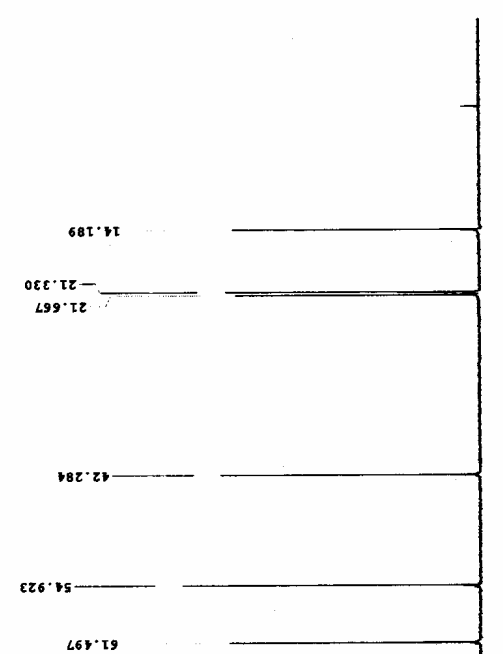
悬

i

ㅇ

:

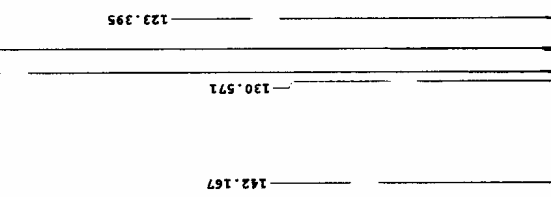

TLS. $\tau L \tau-$ $6 r<\cdot \tau L \tau$ 
${ }^{1} \mathrm{H}$ NMR (400MHz, $\left.\mathrm{CDCl}_{3}\right)$
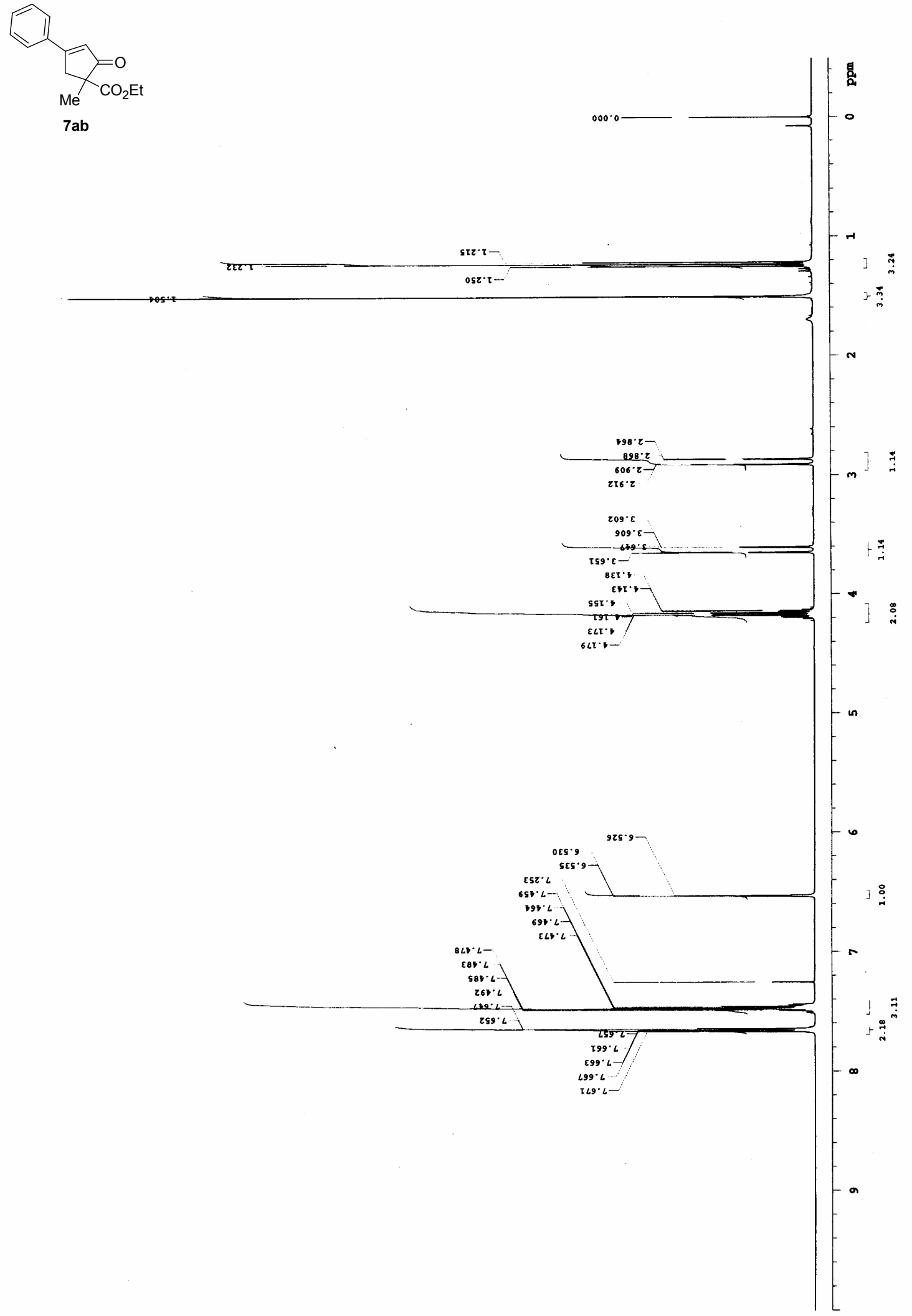
${ }^{13} \mathrm{C}$ NMR (100MHz, $\left.\mathrm{CDCl}_{3}\right)$

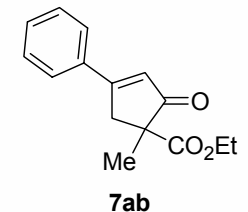

ع89.9L-.

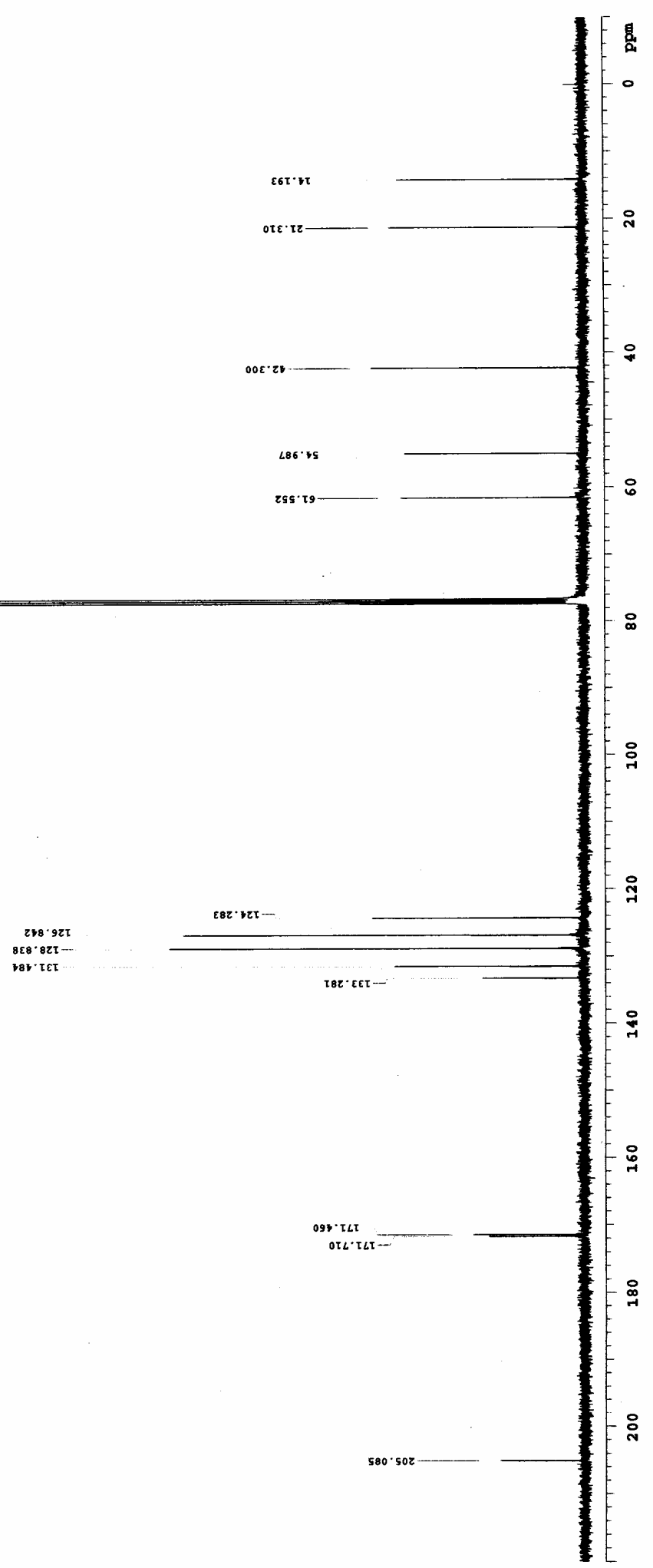


${ }^{1} \mathrm{H}$ NMR (400MHz, $\mathrm{C}_{6} \mathrm{D}_{6}$ )
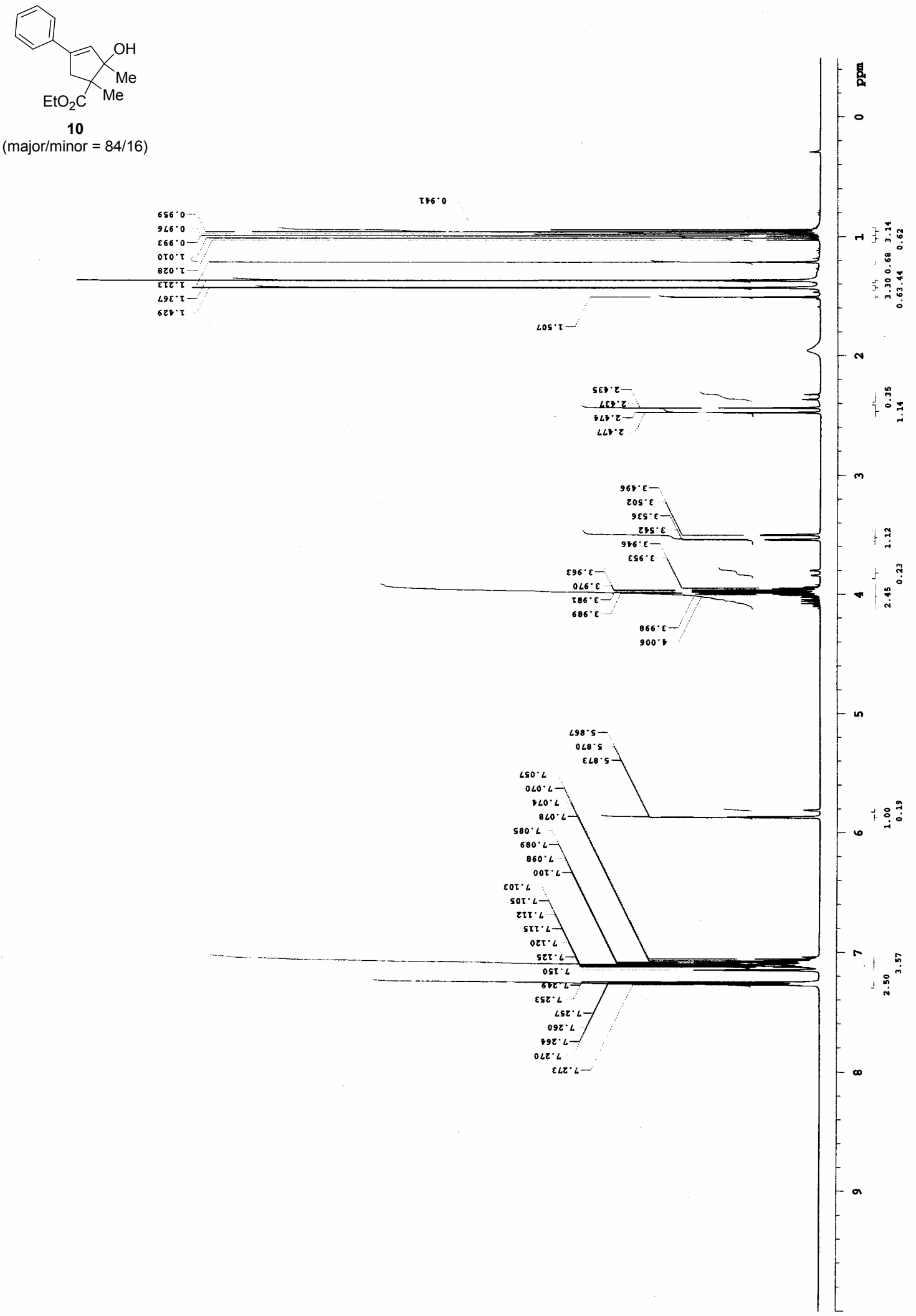
${ }^{13} \mathrm{C}$ NMR (100MHz, $\left.\mathrm{C}_{6} \mathrm{D}_{6}\right)$
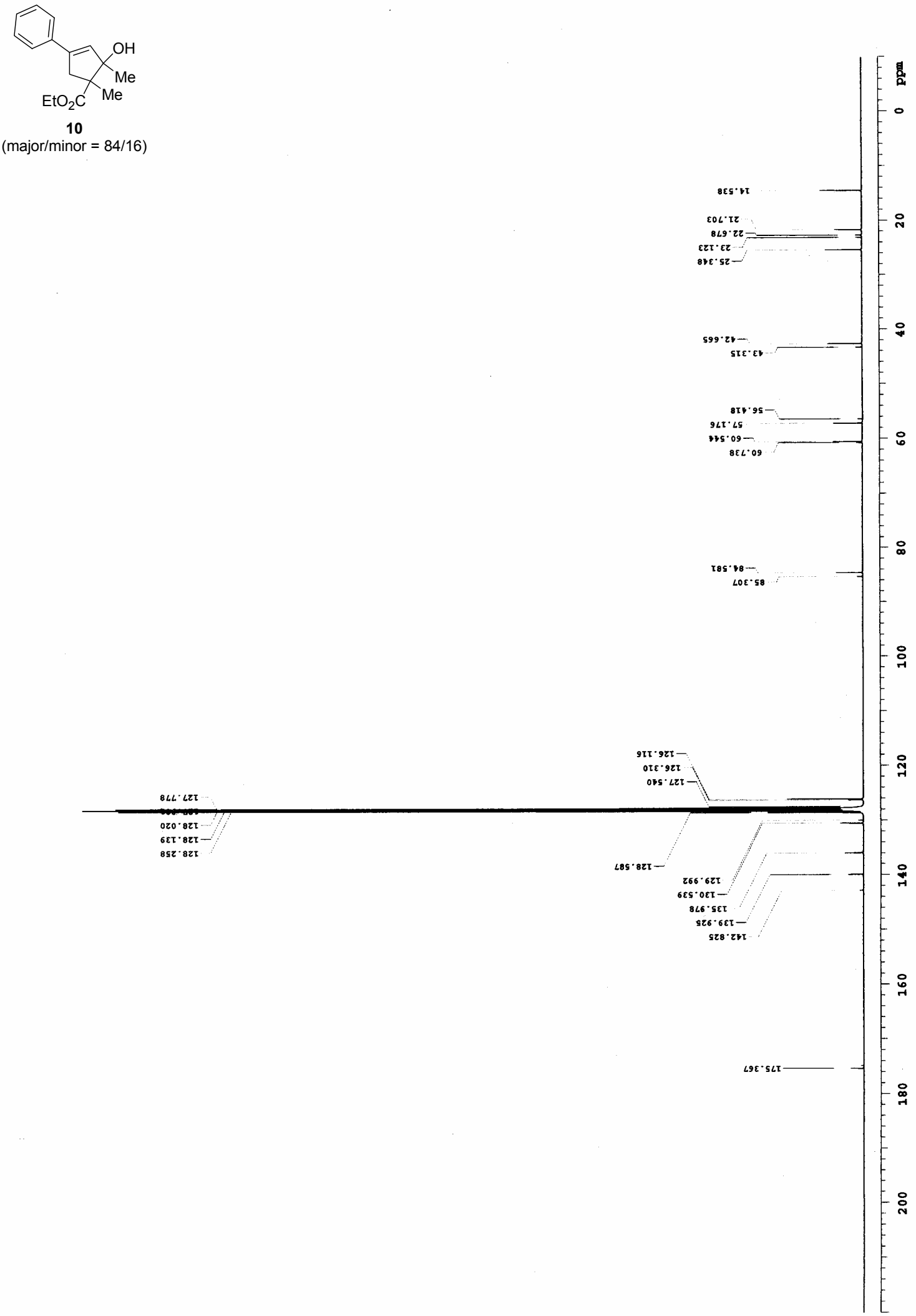
${ }^{1} \mathrm{H}$ NMR $\left(400 \mathrm{MHz}, \mathrm{C}_{6} \mathrm{D}_{6}\right)$

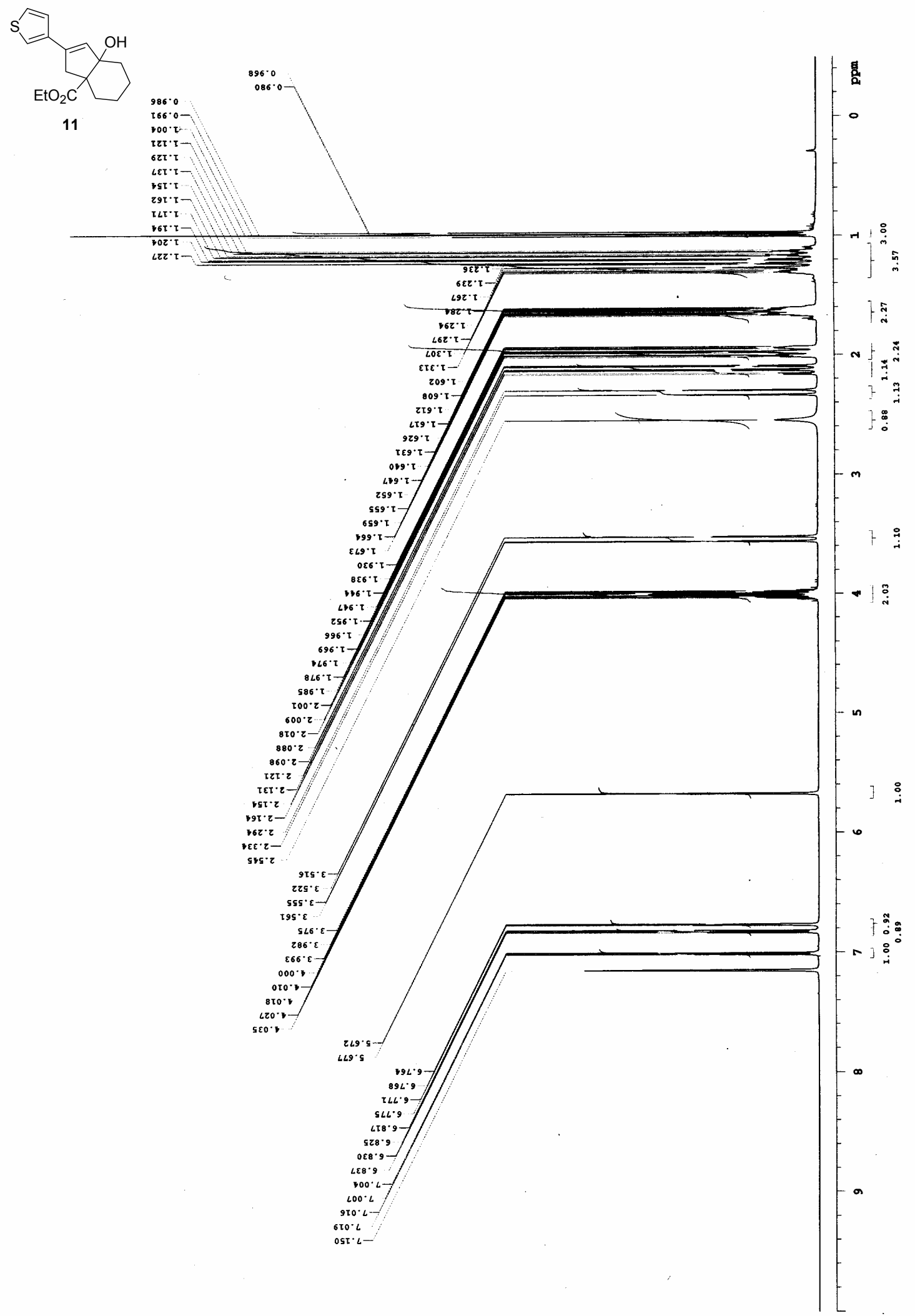


${ }^{13} \mathrm{C}$ NMR (100MHz, $\left.\mathrm{C}_{6} \mathrm{D}_{6}\right)$

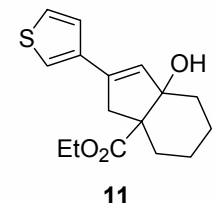

11

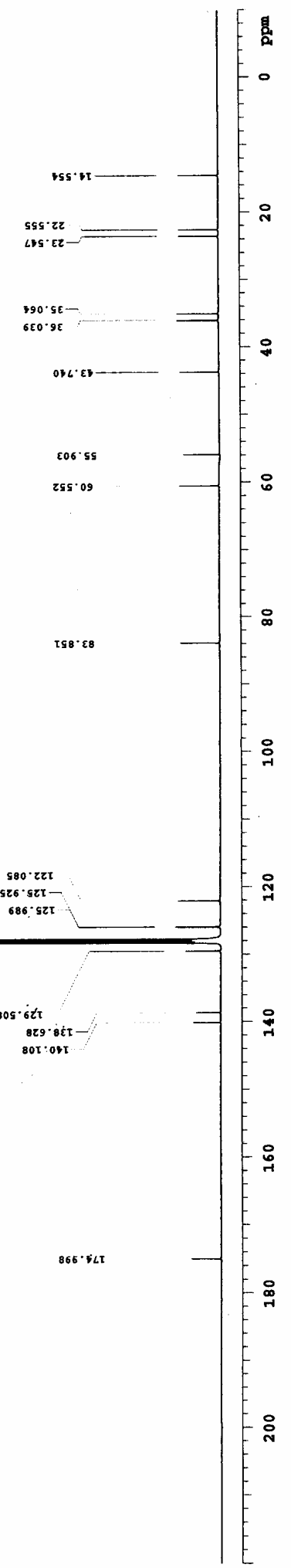

